\title{
Multiplexed Nanobiosensors: Current Trends in Early Diagnostics
}

\author{
Greta Jarockyte ${ }^{1,2} \mathbb{D}$, Vitalijus Karabanovas ${ }^{1,2, *(\mathbb{D}}$, Ricardas Rotomskis ${ }^{2} \mathbb{D}$ and \\ Ali Mobasheri 1,3,4
}

1 Department of Regenerative Medicine, State Research Institute Centre for Innovative Medicine, Santariskiu 5, LT-08406 Vilnius, Lithuania; greta.jarockyte@imcentras.lt (G.J.); ali.mobasheri@imcentras.lt (A.M.)

2 Biomedical Physics Laboratory, National Cancer Institute, Baublio 3b, LT-08406 Vilnius, Lithuania; ricardas.rotomskis@nvi.lt

3 Research Unit of Medical Imaging, Physics and Technology, Faculty of Medicine, University of Oulu, FI-90014 Oulu, Finland

4 Departments of Orthopedics, Rheumatology and Clinical Immunology, University Medical Center Utrecht, 3508 GA Utrecht, The Netherlands

* Correspondence: vitalijus.karabanovas@nvi.lt

Received: 9 November 2020; Accepted: 30 November 2020; Published: 2 December 2020

\begin{abstract}
The ever-growing demand for fast, cheap, and reliable diagnostic tools for personalised medicine is encouraging scientists to improve existing technology platforms and to create new methods for the detection and quantification of biomarkers of clinical significance. Simultaneous detection of multiple analytes allows more accurate assessment of changes in biomarker expression and offers the possibility of disease diagnosis at the earliest stages. The concept of multiplexing, where multiple analytes can be detected in a single sample, can be tackled using several types of nanomaterial-based biosensors. Quantum dots are widely used photoluminescent nanoparticles and represent one of the most frequent choices for different multiplex systems. However, nanoparticles that incorporate gold, silver, and rare earth metals with their unique optical properties are an emerging perspective in the multiplexing field. In this review, we summarise progress in various nanoparticle applications for multiplexed biomarkers.
\end{abstract}

Keywords: multiplexing; nanoparticles; quantum dots; gold nanoparticles; silver nanoparticles; upconverting nanoparticles

\section{Introduction}

The clinical diagnosis of many diseases depends on the accurate and unambiguous detection of various biomarkers, which may include proteins or other biomolecules [1-3]. Immunoassays are a well-established tool for measuring analytes that are normally present at very low concentrations and cannot be determined accurately by other less expensive tests. Standard immunoassays are used to detect one specific analyte, however, for complex diseases such as cancer, diabetes, rheumatoid arthritis (RA) and osteoarthritis (OA), which involve a multitude of biomarkers, one analyte is not enough to obtain an early and accurate diagnosis. In this case, multiple immunoassays can be employed. However, immunoassays require samples that are collected, labelled, archived, and bio-banked according to established laboratory protocols before even conducting the assays, which makes this method challenging and time-consuming. In order to diagnose at the earliest stages of disease development, it is crucial to detect as much information as possible from small quantities of clinical samples. In this context, multiplexed immunoassays are an obvious and attractive approach, especially when sample volumes are limited. The main advantage of multiplexed analysis is the capability to detect multiple 
biomarkers qualitatively or quantitatively in a single sample. There are additional advantages to this approach, such as more data points from single samples, reduced cost per data point, fewer errors due to fewer samples, and increased throughput (Figure 1). Conversely, cross-reactivity remains a significant challenge. If antibodies cannot distinguish between the analyte and other structurally similar components, the specificity of immunoreactivity drastically decreases, and as a consequence, multiplexed detection may not work properly in complex biological and clinical samples.

Traditional diagnostics

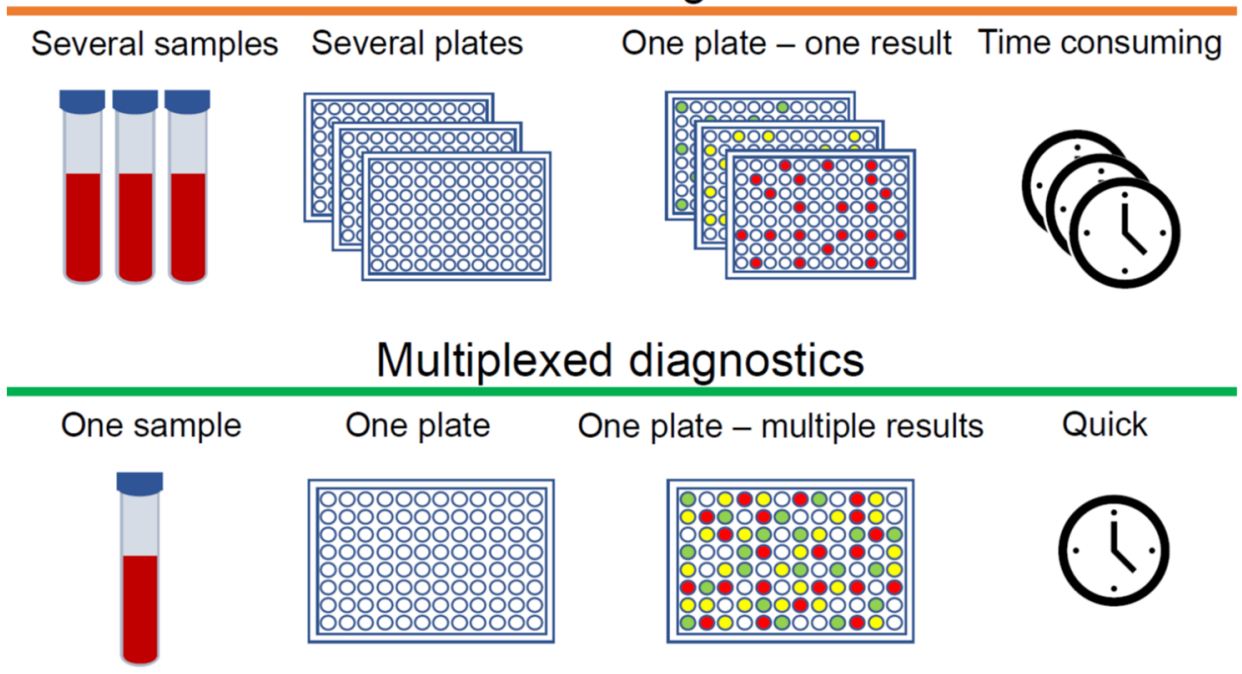

Figure 1. Differences between traditional and multiplexed diagnostics.

Through advances in nanomedicine, as well as progress in areas such as nanoelectronics, nanomaterials, and optics, there has been a steady evolution in the development of miniaturised nanobiosensors for performing biological analysis based on a variety of multiplexing technologies. Nanobiosensors are sensors in which various nanosize materials are used, however, large equipment is still required for whole sensing systems, because they are not, by themselves, able to detect nanoscale events. The current state-of-the-art nanobiosensors are based on several diagnostic methods, such as enzyme-linked immunosorbent assay (ELISA), flow cytometric immunoassay, electrochemical (amperometric, voltammetric and impedance), immunoassay, mass spectrometric immunoassay, chromatographic immunoassay, and different optical immunoassays. Contemporary multiplexed nanobiosensors are developed for the detection and quantification of clinically relevant analytes in biological and clinical samples (i.e., blood, urine, and saliva).

However, in order to successfully implement multiplexed biosensing systems, several crucial elements are required [4,5]. The two key steps in any immunoassay are efficient capture and specific detection. Firstly, recognition probes (e.g., antibodies and other biomolecules), that can interact with specific analyte are required. Specific antibodies can recognize analytes, however for the detection of immunoreactivity, additional elements are needed. Thus, a second and crucial factor is required-the detection probe. For this purpose, stable optical markers or labels that can provide the signal contrast in the final imaging process can be used. These probes with bio-functional groups provide optical signals to confirm and quantify the specific analytes. The last element is a readout system, which can evaluate and quantify detection probes, thus providing information about specific analytes. Signals for readout are usually based on fluorescence parameters such as wavelength, intensity, and lifetime. Although a variety of different fluorescent dyes and fluorophores have been used as detection probes in various immunoassays, only a few fluorescent probes are capable of monitoring multiple analytes in bioassays with distinct optical signals. Compared to fluorescent dyes, nanoparticles are stable, bright fluorophores with high fluorescence quantum yields, narrow fluorescence emission bands (quantum dots and upconverting nanoparticles), larger Stokes shift, long fluorescence lifetimes, 
high resistance to photobleaching, and can provide excitation of several different emission colours (quantum dots) using a single wavelength for excitation. In this review, we summarize progress in nanomaterial-based multiplexed bioassays, by focusing on their applications in medicine.

\section{Quantum Dots}

Since their first description in a biological context [6] quantum dots (QDs) have been considered one of the most attractive luminescence label in biomedicine. Quantum dots are inorganic semiconductor crystals with a diameter of $1 \mathrm{~nm}$ to $10 \mathrm{~nm}$ (typically 2-6 nm), with optical properties which arise from interactions between electrons, holes, and their local environments [7,8]. QDs absorb photons whose energy exceeds the photoluminescence excitation bandgap. In this process, electrons are transferred from the valence band to the conduction band. Due to many electronic states, QD photoluminescence excitation spectra are wide, but on the other hand, QD emission bands are narrow and symmetrical. These unique properties allow different QDs to be excited by the same light source and obtain different coloured visualisations in one biological sample. For comparison, the majority of organic fluorescent dyes have relatively narrow excitation bands and wide fluorescent band. Therefore, in order to perform multicolour imaging, several different light sources are required [8]. By varying the size and composition of QDs, the emission wavelength can be tuned from blue to near-infrared [8]. Additionally, because the surface of QDs are easily modifiable, it is easy to attach various antibodies and other biomolecules to them [9]. Thus, QDs are highly applicable for multiplexed immunoassays. Here, we briefly review how QDs could be used for various multicolour biosensing applications.

\subsection{QLISA}

Quantum dot-linked immunosorbent assay (QLISA) is an approach similar to the enzyme-linked immunosorbent assay (ELISA), except QDs are used instead of enzymes [10]. The principle of these two methods is the same. For both methods, the first step is antibody coating-the specific capture antibody is immobilised on high protein-binding plates. When the capture antibody is immobilised, the uncovered space on the plate is blocked with an irrelevant blocking protein, e.g., serum albumin. After that, samples and standard dilutions are added to the wells. In this step, the analyte adheres to the capture antibodies. In some ELISA cases, analytes can be attached directly on the plate surface, without using a capture antibody. The excess sample is removed, and then specific biotinylated detection antibody is added to the wells to enable detection of the captured protein. In the case of QLISA, QDs with conjugated detection antibody are used. After detection, the antibody binds to the analyte, and streptavidin conjugated with alkaline phosphatase/horseradish peroxidase (ELISA) or streptavidinated QDs (QLISA) is added to the wells which binds to the biotinylated antibody. For the ELISA method one addition step is required before analysis-addition of colorimetric substrate which form a coloured solution when catalysed by the enzyme. Samples are then analysed with microplate readers. The optical density in each well of the multi-well plate is measured, whereas for QLISA, photoluminescence of the QDs is registered.

ELISA is an established standard biomarker detection method that is widely used in biomedical research and in the clinical diagnostic setting [2,11], whereas QLISA is still in the development stage. Nevertheless, the ELISA method still has some important disadvantages, which hopefully QLISA could solve. The main drawback of the conventional ELISA method is that the detection limit is barely less than the nanomolar concentration level, which is usually not enough to reach the clinical threshold of many protein biomarkers, especially in the early stages of disease [12]. This disadvantage is due to low signal-to-noise ratio and limited signal amplification [13]. An additional drawback is that traditional ELISA detects only one analyte in a sample, and multiplexed detections require several measurements simultaneously carried out [14]. These disadvantages can be solved by using luminescent QDs instead of enzymatic colorimetric reactions for identification of the detection antibody. First of all, photoluminescence-based assays are more sensitive than absorbance-based assays [15], thus the signal-to-noise ratio increases by measuring photoluminescence instead of absorption. 
Moreover, QDs have a high photoluminescence quantum yield, which allows acquisition of high signal intensities from relatively low concentrations [16]. Therefore, QDs could work as amplifiers when low concentrations of analyte need to be detected. Finally, QDs, due to their optical and chemical properties, are good candidates for use in multiplexing (Figure 2).

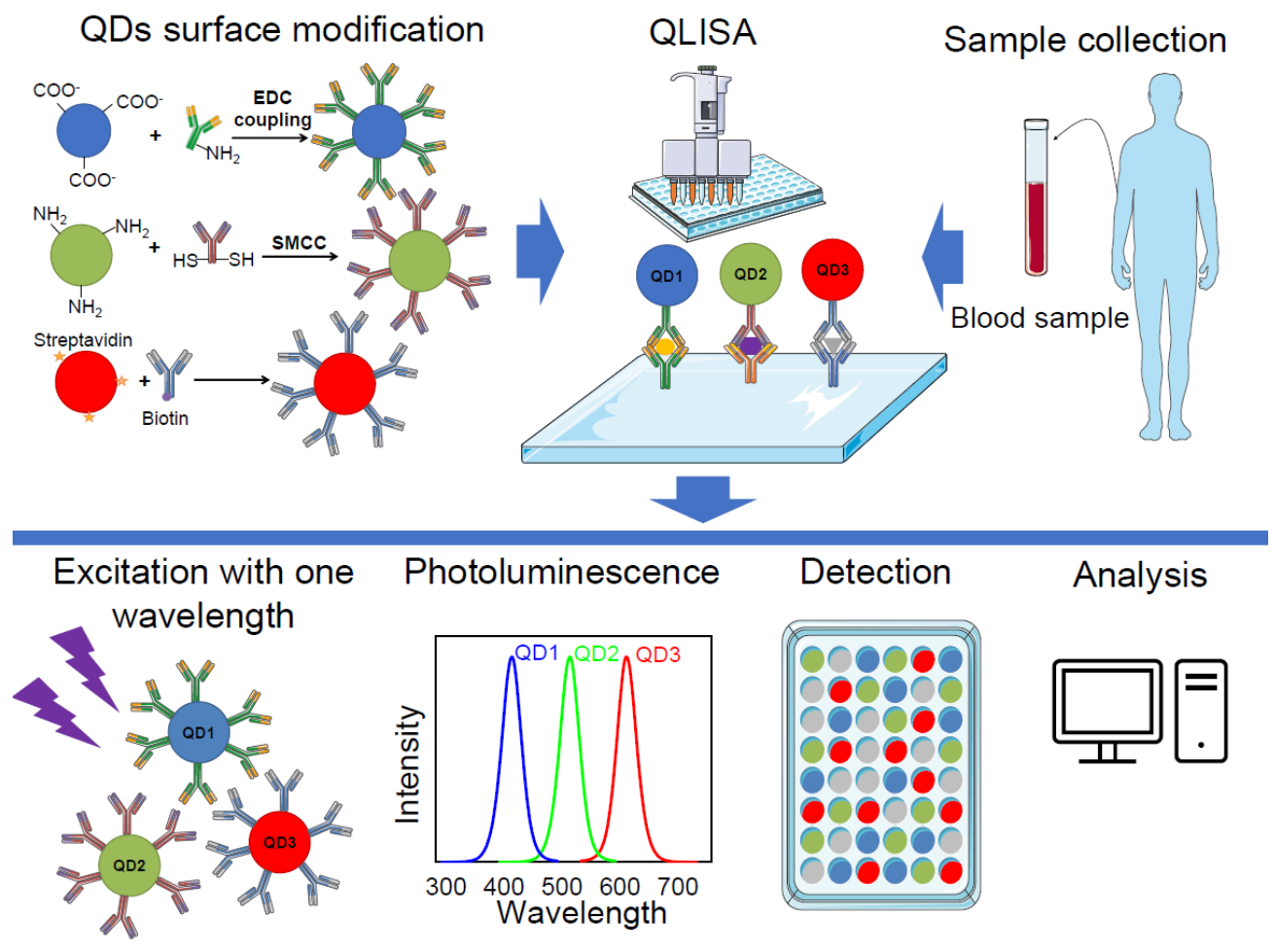

Figure 2. Multiplexed diagnostics using quantum dot-linked immunosorbent assay (QLISA). Firstly, the surface of the quantum dots (QDs) is modified with specific antibodies using 1-Ethyl-3-(3-dimethylaminopropyl)carbodiimide (EDC) coupling, succinimidyl-4-(N-maleimidomethyl) cyclohexane-1-carboxylate (SMCC) conjugation, or streptavidin-biotin interaction. Clinical samples from patients are processed and loaded to a QLISA plate, which is coated with capture antibodies. Then, modified QDs are loaded and the plate is scanned using a microplate reader. Different QDs in the sample are excited with the same wavelength and several bands of photoluminescence are detected. The final step is the analysis of the acquired data.

Mansur et al. (2015) successfully demonstrated the detection of the cluster of differentiation 20 (CD20) antigen, which is a biomarker overexpressed by B-lymphocyte cancer cells, using QLISA [17]. Suzuki et al. (2017) reported the application of QLISA for the detection of the multifunctional cytokine interleukin 6 (IL-6) which is involved in many cellular processes, such as receptor synthesis, inflammation, cell proliferation, and cancer cell signalling [10]. Functionalised QDs were used in QLISA to detect and quantify IL-6. Results demonstrated that the lower limit of IL-6 detection would be approximately $50 \mathrm{pg} / \mathrm{mL}$, which is undetectable using a standard ELISA method [10]. However, these two studies demonstrated working QLISA for the detection of only one biomarker. Nevertheless, the strategies that they described could also be applied for multiplexed QLISA by increasing the number of different QDs-antibody conjugates to detect more than one biomarker.

The first multiplexed QLISA was described by Goldman et al., published in 2004 [18]. In this study, four different toxins (cholera toxin, ricin, shiga-like toxin 1, and staphylococcal enterotoxin $B$ ) were simultaneously detected using CdSe/ZnS QDs (510, 555, 590, and $610 \mathrm{~nm}$, respectively) bioconjugates. The detection of the four analytes was demonstrated at 1000 and $30 \mathrm{ng} / \mathrm{mL}$ of each toxin in the mixture. In their study, Goldman et al. demonstrated the principle of multiplexed QLISA and proposed that their work highlights the advantages of QDs versus conventional organic dyes for use in multiplexing 
applications. However, additional studies are clearly necessary to optimise assay conditions and antibody reagents to produce a reliable and robust multiplexed assay [18].

Song et al. (2015) demonstrated the application of QLISA for the detection of multiplexed residues of antibiotics in milk [19]. A CdTe QDs detection probe conjugated with different antibodies against antibiotics was used. The limit of detection of this assay is $5 \mathrm{pg} / \mathrm{mL}$, thus the developed method showed excellent sensitivity and specificity for the target analytes. Moreover, this assay is quicker than standard ELISA and can be performed in $90 \mathrm{~min}$. Although the authors focused on developing multiplexed nanobiosensors for food safety control, Song et al. developed a method that can easily be adapted for the purpose of various biomarkers detection.

\subsection{Magnetic Bead-Quantum Dot Assay}

Magnetic beads are usually used for the extraction or purification of biomolecules such as proteins, antibodies, and nucleic acids. Bio-functionalised magnetic beads are widely used in cell sorting, bioseparation, and immunoassays. Functionalisation of magnetic beads with specific antibodies leads to decent recognition, separation, and collection of biomarkers in liquid biopsies without additional separation procedures. High surface-to-volume ratios of magnetic beads enables the capture of analytes in a low concentration solutions [20].

The first report of a multiplex magnetic bead assay was in 1977 [21]. This was an immunoassay that used magnetic beads to simultaneously measure multiple analytes in a clinical sample. A multiplex magnetic bead assay is a derivative of a traditional ELISA, using beads for binding the capture antibody, which are available in several different formats based on the utilisation of flow cytometry, chemiluminescence, or electrochemiluminescence technology [14,22]. As mentioned earlier, a traditional ELISA detects only one analyte in a sample, whereas this method was developed with the purpose of measuring multiple analytes in the same sample at the same time. Confusion can be caused by the fact that this method has been described in various ways in the literature, as multiplex bead array assays [14], Luminex multi-analyte or Luminex assay (trademark of Luminex company), multiplex ELISA [23], magnetic immunoassay [24], flow cytometric multiplex arrays or bead-based multiplex assays [22], or a cytometric bead array system (product of BD Biosciences). All the methods mentioned in this context use different antibodies for efficient capture of analytes, while magnetic beads separate target biomolecules from other molecules in the clinical specimens. Nonetheless, bioassays differ according to the optical detection systems used and the different terminologies used in the scientific publications.

There are several multiplex magnetic bead assays, which are different from each other only by virtue of small modifications. For clarity, we will shortly explain the most commonly used method-the Luminex assay. This immunoassay technique is the ELISA with flow cytometry [25]. For this assay, paramagnetic microspheres or beads that are internally labelled with different concentrations of fluorophores (red and infrared) and conjugated to analyte-specific capture antibodies are used. Each fluorophore combination corresponds to one analyte. A mixture of colour-coded beads is added to the sample (or vice versa) and the antibodies bind to the analytes of interest. By completion of this procedure, the additional complementation of biotinylated detection antibodies leads to the formation of an antibody-antigen sandwich. At this point, fluorophore (commonly phycoerythrin (PE)) labelled streptavidin is added, which binds to the biotinylated detection antibodies. For detection, a dual-laser flow-based detection instrument is used for bead classification, analyte determination, and amount of analyte evaluation. This assay can identify multiple biomarkers in biofluid samples and precisely quantify them [25].

Magnetic bead-quantum dot assay is a similar detection method, except instead of organic dyes, quantum dots are used (Figure 3). There are several articles that have established magnetic bead-quantum dot assays to detect one specific analyte [20,26-28]. The most sensitive method using a magnetic bead-quantum dot sandwich assay for the capture and detection of human S100B protein (most extensively studied biomarker for mild traumatic brain injury) in serum was demonstrated 
by Kim et al. in 2015 [20]. Kim et al. have demonstrated that their method provides highly specific detection of S100B protein - with a detection limit of $10 \mathrm{pg} \mathrm{mL}^{-1}$-and a 3 order of magnitude of the detection range up to $10 \mathrm{ng} \mathrm{mL} \mathrm{m}^{-1}$. The overall assay time is $1 \mathrm{~h}$, making this method relatively fast and sensitive [20].
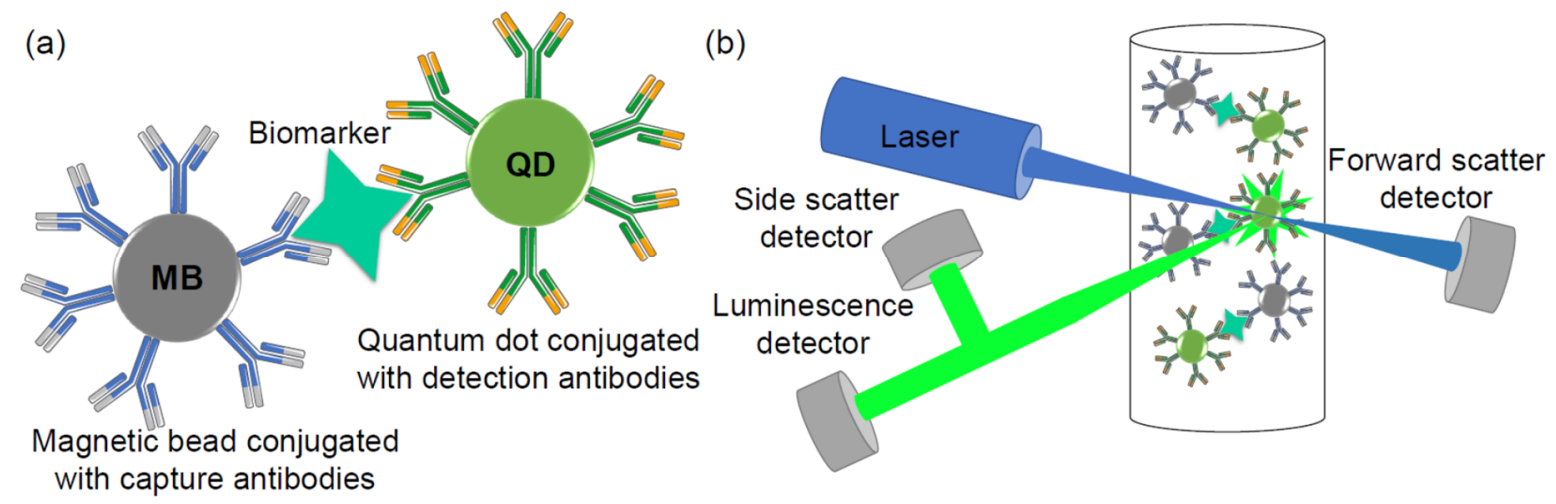

Figure 3. Schematic illustration of a magnetic bead-quantum dot (MB-QD) sandwich assay for biomarker capture and detection; (a) magnetic bead-biomarker-quantum dot conjugate; (b) simplified flow cytometer scheme.

In 2017, the same group developed a droplet-based device for simple, fast, and cheap malaria biomarker PfHRP2 detection [29]. This method was designed by adapting a magnetic bead-quantum dot assay to vial-based assay. This detection tool allows for quantitative and sensitive evaluation of the target biomarker [29].

Liu et al. (2016) proposed multiplex magnetic bead-quantum dot assay in microarray format to detect lung cancer biomarkers [30]. The $6.5 \mu \mathrm{m}$ diameter magnetic beads and QDs (525 nm, $585 \mathrm{~nm}$ and $625 \mathrm{~nm}$ ) both conjugated with antibodies against cytokeratin-19 fragment (CYRFA 21-1), neuron specific enolase (NSE) or carcinoembryonic antigen (CEA) lung cancer biomarkers were added into human serum samples and mixed in a test tube, and were introduced onto the array of the chip. Microbead-antigen-QD conjugates were detected by fluorescent microscope, equipped with a charge-coupled device (CCD) camera. Results showed that this sandwiched immunoassay successfully detects the presence of different lung cancer associated biomarkers such as CYRFA 21-1, NSE and CEA in the given biological fluid at low concentrations (detection limit $0.97 \mathrm{ng} / \mathrm{mL} ; 0.37 \mathrm{ng} / \mathrm{mL} ; 0.19 \mathrm{ng} / \mathrm{mL}$, respectively). The authors suggest that this method could be a low cost tool for disease diagnosis [30]. In later work, the same group demonstrated a similar approach focusing on the same three lung cancer biomarkers, using the multiplexed detection and micro-magnetic beads as immune carriers and QDs as detection probes [31]. Immunocomplexes were visualised using fluorescence microscopy and the micrographs were analysed by image analysis software to quantify the luminescence of QDs. Using this method, the authors managed to reach lower than $1 \mathrm{ng} / \mathrm{mL}$ detection limit (364 pg/mL for CYRFA 21-1, $38 \mathrm{pg} / \mathrm{mL}$ for CEA, $370 \mathrm{pg} / \mathrm{mL}$ for NSE) [31].

Bai et al. (2019) used bead-based microarray to detect three lung cancer biomarkers (CEA, CYFRA 21-1 and ProGRP) from exosomes [32]. By using a microfluidic system, exosomes were collected from cell culture supernatant or plasma samples from lung cancer patients. After the isolation of exosomes, tumour biomarkers were detected by using QDs conjugated with detection antibodies. The difference between experimental result and clinical data was minimal, thus this method could be applied for clinical testing [32].

$\mathrm{Li}$ and co-workers developed barcodes for multiplexed detection using magnetic beads and QDs [33,34]. In 2016 they introduced barcodes for the multiplexed detection of five different tumour biomarkers in serum (AFP, CEA, CA199, CA125, and CA242). Cadmium-free NIR-emitting CuInS $2 / Z n S$ QDs and superparamagnetic $\mathrm{Fe}_{3} \mathrm{O}_{4}$ in PSMA microspheres were used for simultaneous biomarkers detection. Using these barcodes, they detected sub-ng/mL concentrations of analytes [33]. 


\subsection{Multiplex Flow Cytometric Immunoassay}

Magnetic beads are convenient for immunoassays, due to the possibility of controlling them with a magnetic field and separating them from the mixture when it is needed. However, non-magnetic beads are more frequently used for multiplexed flow cytometric immunoassays.

Yu et al. (2012) have developed a competitive microbead-based flow cytometric immunoassay for the simultaneous detection of two biomarkers using QDs luminescent labels [35]. The purpose of their study was the development of a duplex detection system for the detection of toxins (microcystin-LR, benzo[a]pyrene), which can be found in drinking water as contaminants. These two molecules are too small to apply a sandwich immunoassay, thus a competitive immunoassay was designed to determine molecules using a single antibody. During competitive reaction, antigen competitors on polystyrene beads are changed with target molecules. Then, QDs conjugated with antibodies against the target molecules are added to the solution. The final stage of the assay is photoluminescent measurement of the sample without any wash step, using a flow cytometer. QD-antibody conjugates label both free and polystyrene bead attached antigens, although during flow cytometer analysis the groups are easily separated. This method allows not only qualitative, but also quantitative analysis. Microcystin-LR detection dynamic range was $0.52-30 \mu \mathrm{g} \mathrm{L}^{-1}$ and for benzo[a]pyrene it was $0.13-10 \mu \mathrm{g} \mathrm{L}^{-1}$. The proposed assay was performed within $30 \mathrm{~min}$, thus this is a relatively fast method for multiple analyte detection [35].

Bilan et al. (2017) demonstrated the detection of multiple lung cancer biomarkers using microspheres encoded with QDs (QDEMs) in clinical samples of bronchoalveolar lavage fluid [36]. In their study, 4.08, 6.1 and $8.24 \mu \mathrm{m}$ carboxylated melamine formaldehyde resin microspheres as matrix cores and CdSe/ZnS QDs $\left(\lambda_{\mathrm{em}}=515 \mathrm{~nm}\right)$ as optical codes for the preparation of QDEMs were used. Antibodies against three lung cancer biomarkers (AMBP, PRDX2, and PARK7) were conjugated with different sized QDEMs. Using QDEM-antibody conjugates, all three lung cancer biomarkers were successfully and simultaneously detected in clinical samples using a flow cytometer. The authors compared their method's reproducibility and reliability with the commonly used Luminex $\mathrm{xMAP}^{\circledR}$ bead-based immunoassay. Their results showed that QDEM technology may be considered as an alternative to Luminex $x M A P^{\circledR}$ for the diagnostic purpose using conventional flow cytometers. However, the authors did not achieve a high analytical sensitivity at low concentrations of the biomarkers, thus the sensitivity of this method remains unknown [36].

The main issue with the flow cytometry method is difficulties in achieving nanoparticles of homogeneous size. Heterogeneity of nanoparticles leads to interference due to morphology dependant on Rayleigh scattering signals.

\subsection{Electrochemical Immunoassay}

Electrochemical immunoassay is based on a solid phase system where an antibody-antigen reaction occurs and an electrochemical detection system is integrated in the same device [37]. In this method, electrochemical sensors are physically attached to the detection probe surface. When the detection probe interacts with the target analyte, a measurable electrochemical signal appears. These biosensors have impressive detection characteristics and are recognised as one the best sensing systems [37].

The first utilisation of semiconductor nanoparticle labels for this method was demonstrated for the electrochemical DNA hybridisation assay [38,39]. Since then, only a few papers have described how quantum dots could be used in multiplexed electrochemical immunoassay. A multiplex electrochemiluminescence immunoassay has been developed for the simultaneous determination of two different tumour biomarkers (alpha-fetoprotein (AFP) and CEA) in human serum and saliva, using multicolour QD labels and graphene as a conducting bridge [40]. Streptavidin-coated CdSe/ZnS (525 nm and $625 \mathrm{~nm}$ ) were conjugated with biotin-labelled secondary antibodies (anti-AFP 2 and anti-CEA respectively). QDs conjugated with secondary antibodies produced electrochemiluminescence reactions after the immunoreaction and the intensity of electrochemiluminescence was amplified using graphene as a conducting bridge. The quantity of AFP and CEA was indicated by the electrochemiluminescence 
responses of $\mathrm{QDs}_{525}$ and $\mathrm{QDs}_{625}$, respectively. The working range of the proposed method was $0.001-0.1 \mathrm{pg} / \mathrm{mL}$ and the detection limit was $0.4 \mathrm{fg} / \mathrm{mL}$ for both analytes [40].

Ultrasensitive electrochemiluminescence immunoassays have been developed for the simultaneous determination of two biomarkers, the cancer antigens CA 125 and CA 15-3. The immunosensor designed in this study can be used for the sequential detection of CA 125 and CA 15-3 biomarkers with the wide detection ranges of $1 \mu \mathrm{U} / \mathrm{mL}-1 \mathrm{U} / \mathrm{mL}$ and $0.1 \mathrm{mU} / \mathrm{mL}-100 \mathrm{U} / \mathrm{mL}$ with very low detection limits of $0.1 \mu \mathrm{U} / \mathrm{mL}$ and $10 \mu \mathrm{U} / \mathrm{mL}$, respectively [41].

\subsection{Multiplex Antigen Imaging in Cells and Tissues}

Conventional immunohistochemistry (IHC) is widely used as a diagnostic technique in the field of pathology, especially in cancer diagnostics. However, the standard IHC method allows the labelling of a single marker per tissue section, which is insufficient in some cases, thus multiple sections of same tissue are stained and examined, which is time and resource consuming. The use of several QDs conjugated with specific antibodies enables the easy labelling of multiple biomarkers in one specimen and the distinguishing of cancer cells from healthy cells (Figure 4).
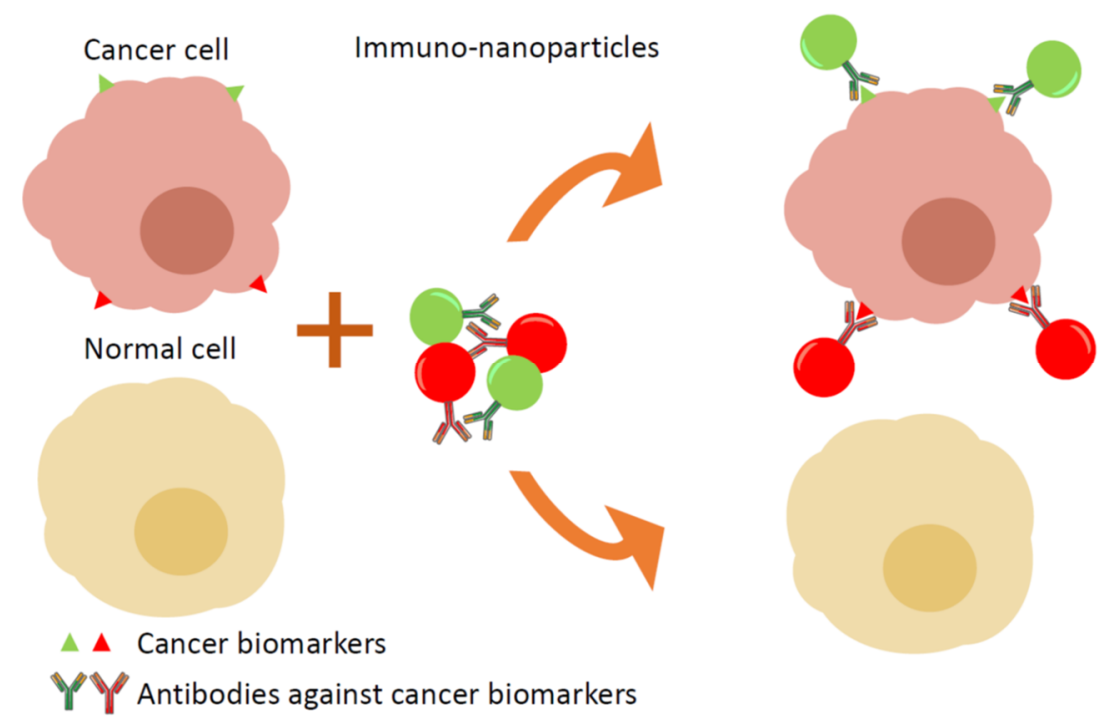

Figure 4. Targeting of cancer cells in tissue samples: multiple immuno-nanoparticles with specific antigens against cancer biomarkers label cancer cells. Each antibody is conjugated with different coloured luminescent nanoparticles, which allows the simultaneous detection of several biomarkers in one sample.

The first report of QD-based imaging of biomarkers was published by Wu et al., 2002 [42]. The authors used CdSe/ZnS QDs $\left(\lambda_{\text {ex }} 535 \mathrm{~nm}\right.$ and $\left.630 \mathrm{~nm}\right)$ to simultaneously label two breast cancer biomarkers in SK-BR-3 cells (cell surface antigen Her2 and nuclear antigens). They demonstrated that QDs were effective for the two-colour fluorescence labelling of distinct cellular components [42].

Yezhelyev et al. published a breakthrough multiplex cell imaging article in 2007 [43]. They demonstrated the quantitative and simultaneous profiling of five biomarkers in breast cancer cells and clinical tissue specimens, using QDs for labelling. Five different QDs (QD525, QD565, QD605, QD655, QD705) conjugated with antibodies against HER2, ER, PR, EGFR, and mTOR biomarkers, respectively, were used for multiplexed detection of these antigens in breast cancer cells (MCF-7 and BT474 cell lines) and in tumour biopsy specimens. All samples were visualised using confocal microscopy and, additionally, spectroscopic measurements were performed for quantification purposes. The authors concluded that multiplexed detection and quantification of ER, PR, and Her2 biomarkers correlated firmly with the results gained from other traditional methods such as IHC, Western blotting, 
and fluorescence in situ hybridisation, implying that the QD-based sensing technology is applicable for the molecular profiling of tumour biomarkers in vitro [43].

Approaching clinical application, Liu et al. (2010) reported the use of QDs for the detection and characterisation of a class of low-abundant tumour cells in Hodgkin's lymphoma [44]. They developed multiplexed detection of four protein biomarkers (CD15, CD30, CD45, and Pax5) on human tissue biopsies. Immunostaining of tissues was performed in two steps repeating them twice: firstly, mouse and rabbit primary antibodies against Pax5 and CD30 antigens, respectively, were used to bind lymphoma biomarkers. After a washing procedure, a mixture of goat anti-rabbit QD655 and goat anti-mouse QD605 secondary antibody-QD conjugates was applied to indicate the primary antibodies. The same procedure was repeated using primary antibodies for the additional antigens CD45 (rabbit) and CD15 (mouse), followed by using secondary antibody-QD conjugates (goat anti-rabbit QD565 and goat anti-mouse QD525). Using the described immunostaining concept, antibodies from only two animal species are sufficient, so this method is cheaper than standard ones. The results indicated that a distinct QD staining pattern can be used not only to detect Hodgkin's lymphoma, but also differentiate it from benign lymphoid hyperplasia [44].

Xu et al. published a study in 2013 focusing on QD-based IHC imaging for multiplexing cancer biomarker detection on formalin-fixed and paraffin-embedded samples of tissues [45]. In this study, QD-primary Ab conjugates were used for the multiplexed detection of survivin and EF1 $\alpha$ in fixed tissue samples. These two biomarkers have different expression profiles in cells, thus different brightness QDs were used for each analyte. EF1 $\alpha$, a highly expressed housekeeping protein, was conjugated with low intensity QD530, whereas survivin was conjugated with high intensity QD620. Survivin was detected only in the cancerous tissues, but EF1 $\alpha$ was present in both cancerous and surrounding healthy tissues. Quantification results showed that survivin expression level in the cancerous tissue was much higher than $\mathrm{EF} 1 \alpha$, although in the non-cancerous tissues expression levels of these two proteins were similar. Thus, these methods enable cancer diagnosis with high fidelity using two biomarkers [45].

Despite current advances, there are some limitations that need further discussion and evaluation. The main issue is the nonspecific binding of QDs, which reduces the specificity of the methodologies that are being developed. Additionally, there is still a lack of commercially available media for QD fixation, which leads to low stability on prolonged storage and the inability to retain fluorescence in stained specimens.

\subsection{Paper-Based Biosensors}

Paper-based biosensors have emerged as simple, low-cost, quick, and user-friendly diagnostical tools. There are three main types of paper-based biosensors: dipstick tests, lateral flow assays, and microfluidic paper-based analytical devices ( $\mu \mathrm{PAD})[46]$. All three methods have their advantages and disadvantages, for example, the dipstick test is easy and fast, however often inaccurate, and analysis takes more time than the test itself. Lateral flow assays are more sensitive, commonly detecting nanograms per $\mathrm{mL}$, also featuring rapid detection, although excess antibodies can lead to agglutination and inaccurate results. Generally, $\mu$ PAD have inefficient sample consumption and high limits of detection, thus usually $\mu \mathrm{PAD}$ is insufficient for the analysis of low abundance biomarkers present in small concentrations in biological samples. Multiplexed detection using paper-based biosensors is still a challenge itself, although for the past few years the number of publications in this field has been growing. The variety of paper-based biosensors are wide; thus, different nanoparticles could be used as labelling agents. QDs are quite a common choice, but metal nanoparticles, carbon dots, inorganic complexes, etc., could be used as well [47].

A paper-based immunochromatographic dipstick method based on sandwich immunoassay for the two-plex detection of lung cancer biomarkers such as CEA and NSE was developed by Xiao et al. [48]. High-quality carboxylated quantum dot beads were used as detection probes and a custom-made strip reader was developed to read photoluminescence signals for quantification. Under optimal conditions, this immunoassay showed a high detection limit of $37.8 \mathrm{pg} / \mathrm{mL}$ for CEA and $42.6 \mathrm{pg} / \mathrm{mL}$ for NSE and 
was approved in clinical human serums with high sensitivity and specificity. Moreover, the authors' results demonstrated that quantum bead-based immunochromatographic dipstick biosensors could be used as a routine assay for rapid diagnostics of lung cancer [48].

Hydrophobic QD-doped polystyrene nanoparticle-based lateral flow test strips were developed for the multiplexed detection of lung cancer biomarkers CYFRA 21-1 and CEA [49]. Detection of antibodies was based on a standard sandwich immunoassay. Serum samples, collected from patients, were mixed with functionalised QD-doped polystyrene nanoparticles and added onto the test strips. Immunocomplexes were formed and immobilised by the capture antibodies on the test lines. Photoluminescence of QDs was detected by a portable fluorescence strip reader for further analysis. The authors demonstrated a detection range of $1.3-480 \mathrm{ng} / \mathrm{mL}$ for CYFRA $21-1$ and $2.8-680 \mathrm{ng} / \mathrm{mL}$ for CEA. The detection limits for CYFRA $21-1$ and CEA were 0.16 and $0.35 \mathrm{ng} / \mathrm{mL}$, respectively. The overall assay test time was $15 \mathrm{~min}$, making it a rapid, sensitive and user-friendly method [49].

Chen et al. demonstrated the multiplexed detection of CEA and prostate specific antigen (PSA), using a paper-based immunodevice [50]. Sandwich immunoassay was used for the capture and detection of cancer biomarkers. Capture antibodies of CEA and PSA were immobilised on the same zone of the paper-based device. Then, samples of human serum were applied to the device and the biomarkers captured by antibodies. Later, detection antibodies conjugated with CdTe QDs (525 and $605 \mathrm{~nm}$ ) were used. QDs were excited and detected simultaneously under $272 \mathrm{~nm}$ excitation wavelength. The linear response of both biomarkers was in the $1.0-40 \mathrm{ng} / \mathrm{mL}$ concentration range and the detection limits were $0.3 \mathrm{ng} / \mathrm{mL}$ for CEA and $0.4 \mathrm{ng} / \mathrm{mL}$ for PSA [50].

\section{Gold and Silver Nanoparticles}

One of most widely used groups of nanoparticles are synthesised from noble metals, such as gold and silver. Gold (Au) and silver (Ag) nanoparticles (AuNPs and AgNPs) are the most stable metal nanoparticles, and they have numerous attractive features such as size-related electronic, magnetic and optical properties (quantum size effect), large optical field enhancements resulting in the strong scattering and absorption of light, and their perspective applications in biomedical field [51]. Noble metal nanoparticles, which are composed of tens or hundreds of atoms and typically $<2 \mathrm{~nm}$ size, are known as nanoclusters. These nanoclusters have molecule-like properties due to their extraordinary physical and chemical characteristics, and they have especially attracted the attention of scientists [52]. AuNPs are known as non-toxic and biocompatible nanoparticles; thus they are frequently used in studies related to diagnostic tool development, drug delivery, novel therapeutic agents, and other medical applications [53,54]. Whereas, AgNPs have unique antimicrobial features, which allows silver nanoparticles to be used in the treatment of microorganisms such as bacteria, fungi, and viruses [55]. In this article, the application of AuNPs and AgNPs for multiplexed biomarkers detection will be briefly reviewed.

\subsection{Plasmonic Multiplex Sensing}

Gold and silver nanoparticles are exceptional due to their plasmonic properties. Such nanoparticles demonstrate unique absorption and scattering characteristics in the VIS-NIR spectral region due to photon-induced collective oscillation of their surface electrons. This coherent electron oscillation in noble metal nanoparticles is also known as localised surface plasmon resonance (LSPR). Briefly, LSPR occurs when a nanoparticle (bigger than $2 \mathrm{~nm}$ ) is interacting with light. Electromagnetic oscillating fields cause the conduction electrons of a nanoparticle surface to oscillate coherently. A highly localised oscillating electron cloud is created around the nanoparticle, which rapidly decays away, through resonance enhance far-field scattering. The LSPR profile can be described with spectroscopically measured parameters such as shape, position and intensity, which strongly depend on the properties of nanoparticles, such as size, shape, monodispersity, as well as interaction with ligands or surrounding media [56]. 
Huang et al. (2012) developed a multiplexed bioanalytical assay which allows simultaneous detection of human serum specimens infected by Schistosoma japonicum and tuberculosis pathogens without sample pre-treatment. Two different populations of gold nanorods (AuNRs) were modified with antibodies of different antigens. Detection of biomarkers was achieved by registering a shift in the LSPR, using a standard visible/NIR spectrometer. This nanobiosensor was able to identify infected and uninfected samples and provide a semi-quantitative readout [57].

Utilising the LSPR properties of plasmonic metal nanoparticles, a new variation of the conventional ELISA was established, known as plasmonic ELISA (pELISA) [10]. Several studies have demonstrated the application of pELISA for diagnostics of diseases such as prostate cancer [58-60], syphilis [61] tuberculosis [62], hepatitis B [63] and HIV [64]. However, all pELISA systems are designed for one analyte, and despite its great potential there are no multiplexed pELISAs thus far.

\subsection{Multiplexed Colorimetric Detection}

The nanoparticle-based colorimetric assays are one of the most attractive methods for the detection of various biomolecules such as DNA, RNA, enzymes, proteins, and other small molecules. The key parameter for colorimetric sensing is the ability to change colour of colloidal solution due to changes of noble metal nanoparticles size or distance between them. Such colorimetric sensors can be divided into four types: aggregation, etching, growth and nanoenzyme [65]. The most commonly used gold or silver nanoparticle-based sensors are of the aggregation type, in which optical features of solutions change depending on their level of aggregation. During aggregation of nanoparticles, the surface plasmons of the particles couple and their LSPR profile changes. The main advantages of colorimetric assays are that results can be monitored with the naked eye without the need for any instrumentation. Additionally, this method is simple, convenient, and low cost. Most studies have developed colorimetric detection tools for one analyte [66], however, some multiplexed colorimetric assays have been published.

Mancuso et al. used AuNPs and AgNPs for Kaposi's sarcoma associated herpesvirus and Bartonella DNA simultaneous detection [67]. Specific DNA primers, which recognise targeted oligonucleotide sequences, were attached to AuNPs and AgNPs. When targeted oligonucleotides appear in solution, nanoparticles conjugated with primers bond with them and with each other. Consequently, aggregates of AuNPs and AgNPs are formed and the solution changes colour. Changes were identified visually and by measuring the absorption of the solutions. Authors have demonstrated that the proposed method works when AuNPs and AgNPs are mixed in the same solution-both colour change reactions can be seen independently of each other. In this assay, the limit of DNA detection for the AuNPs is $2 \mathrm{nM}$ and for the AgNPs is $1 \mathrm{nM}$ [67].

Heo et al. demonstrated two-plexed colorimetric assay using three types of nanoparticles, functionalised with DNA: gold nanoparticles (AuNP-DNA), silver nanoparticles (AgNP-DNA), and gold nanorods (AuNR-DNA) [68]. The mixture of these nanoparticles is black-coloured, but after targeted analytes are introduced, the colour of the solution changes, based on which type of nanoparticles (or combination) have aggregated. The authors used a modified CMYK (cyan, magenta, yellow, and key (black)) colour model: when one type of nanoparticle aggregates, corresponding colours are detracted from the mixture. For example, when red-coloured AuNPs aggregate due to interaction with targeted analytes, the mixture turns light-green (cyan and yellow combination). Using this approach, the authors successfully demonstrated the detection of thrombin and platelet-derived growth factor (PDGF) in human blood plasma. The sensitivity of this method was not high (the limit of detection of PDGF was $19 \mathrm{nM})$, however, it was possible to visually detect the detection limit and concentration $(20 \mathrm{nM})$ with the naked eye [68].

Different approaches for the multiplexed colorimetric diagnostic detection of cancer was demonstrated by Di et al. [69]. The authors used decorated AuNPs and antibody conjugated exosomes for a nanozyme-assisted immunosorbent assay to detect CD63, CEA, GPC-3, PD-L1 and HER2 exosomal proteins from four cell lines as well as from clinical serum samples. This method allows 
users to differentiate the levels of the various proteins without additional labelling with detection antibodies, enabling the development of a quicker and much simpler testing procedure [69].

\subsection{Multiplex SERS Imaging}

Surface-enhanced Raman scattering (SERS) is a method for precise molecular detection based on the enhanced Raman scattering of biomolecules, which are localised on nanostructured SERS-active gold or silver surfaces. The advantages of this method are very high sensitivity down to the single molecule level, and the sharp molecularly specific spectra. The use of SERS instead of QD labelling for imaging is superior because SERS provides greater sensitivity for molecular analysis [70]. Moreover, the Raman signals exhibit much narrower peaks compared with traditional fluorophores and QDs; generally SERS signals are only 1-2 nm width [71]. Additionally, Raman signals are exceptionally stable and resistant to photobleaching, independently from measurement conditions. Thus, SERS tags are absolutely applicable for the multiplex detection of soluble biomarkers as well as multiplexed SERS-based cellular imaging (Figure 5).

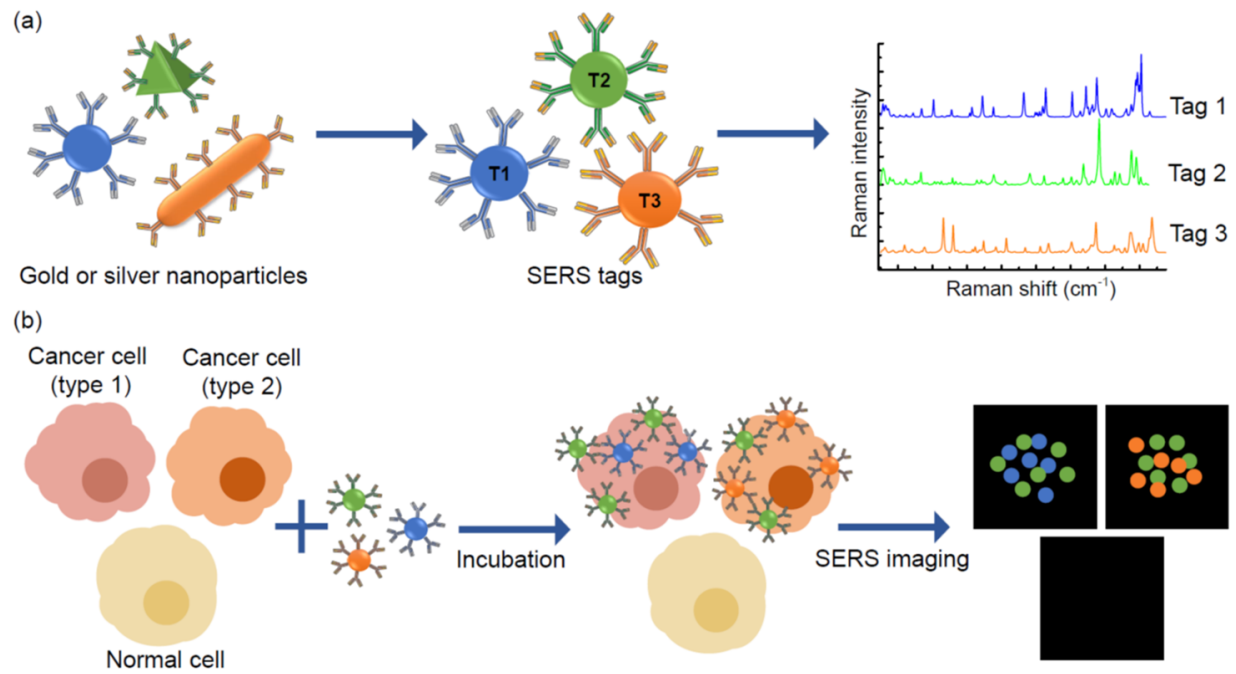

Figure 5. Principles of multiplexed detection using the surface-enhanced Raman scattering (SERS) technique. (a) Noble metal nanoparticles, that are different in size and shape, have unique Raman signals with narrow peaks, thus they could be used as SERS tags. (b) Schematic illustration of simultaneous detection of three tumour associated antigens expressed different types of cancer cells by SERS imaging.

Lee et al. (2012) demonstrated SERS-based cellular imaging technique to detect and quantify multiple breast cancer biomarkers expressed on plasma membranes [72]. Silica-encapsulated AuNPs were conjugated with CD24 and CD44 antibodies and fluorescent dyes (FITC and RuITC), creating dual mode nanoprobes (SERS and fluorescence). They demonstrated that simultaneous detection of CD24 and CD44 biomarkers in breast cancer cells (MDA-MB-231) can be enhanced by combining the two methods: fluorescence imaging for quick detection and SERS imaging for accurate localisation of biomarkers.

Later studies by the same group applied a SERS-based imaging method for the multicolour detection of three breast cancer cell biomarkers [73]. This study was caried out in human breast cancer cell lines (MDA-MB-468, KPL4 and SK-BR-3) by measuring the expression of EGF, ErbB2, andIGF-1 biomarkers. After visualisation, SERS-mapping images were recorded, which allowed them to localise and quantify EGFR, ErbB2 and IGF-1R proteins in cells. Analysis of the experimental results enabled identification and phenotyping of cancer cells [73].

Sun et al. (2015) used polydopamine encapsulated SERS probes for the detection of tumour-associated biomarkers (VEGF, EGFR and Vimentin) in different prostate cancer cell lines (LnCAP, PC-3, DU145) [74]. By using SERS images, the expression of three tumour biomarkers on 
the surface of prostate cancer cells were evaluated visually and three prostate cancer cell lines had been distinguished from each other. The authors suggest that usage of the multiplexed SERS imaging technique could improve the identification of cancer cell phenotypes [74].

Li et al. (2018) developed a multiplexed nanobiosensor by adapting the sandwich-type immunoassay and SERS methods for the detection of cancer biomarkers. Three soluble cancer protein biomarkers (soluble programmed death 1 (sPD-1), soluble programmed death-ligand 1 (sPD-L1) and soluble epithermal growth factor receptor (sEGFR)) were analysed directly from human serum [75]. In this study, for Raman signal improvement, gold-silver alloy nanoboxes were used as SERS tags to facilitate highly sensitive detection. The limit of detection for SPD-1, sPD-L1, and sEGFR achieved by this platform was $6.17 \mathrm{pg} / \mathrm{mL}, 0.68 \mathrm{pg} / \mathrm{mL}$, and $69.86 \mathrm{pg} / \mathrm{mL}$, respectively. They proposed that their nanobiosensor has huge potential in the clinic, because it can accurately and specifically detect several cancer biomarkers in human serum at once [75].

Another study demonstrated multiplex profiling of oestrogen receptor (ER), progesterone receptor (PR), and epidermal growth factor receptor (EGFR) expression in breast cancer tissue and normal tissue sections, using AuNP-based SERS tags [76]. $60 \mathrm{~nm}$ AuNPs with incorporated alkyne and nitrile groups and conjugated to primary antibodies against the growth factors were used as SERS tags. They demonstrated that their SERS nanotags have individual and definite bands in cellular regions without any Raman signal. The method was tested in vitro using the MCF-7 cell line, which expresses ER, EGFR, and PR at high levels and the normal cell line 3T3 in which expression of the biomarkers is downregulated. The results corresponded with data obtained with other methods. Additionally, the authors examined the use of multiple SERS tags for ER, EGFR, and PR imaging in human breast cancer tissue specimens. The results showed higher expression levels of these three biomarkers in breast cancer tissues compared to healthy samples, consistent with the diagnostic and prognostic classification of these tissues. Thus, the authors suggest that their proposed method has potential for multiplex imaging in clinical cancer diagnosis [76].

SERS is a reliable analytical method suitable for the detection of multiple analytes in biological samples even if there are extremely low quantities of target biomarker. By using SERS-nanotags, assays can be conducted directly in biopsy samples as well as in tissues or live cells, if necessary. However, utilisation of noble metal nanoparticles in multiplexed SERS biosensors is not yet cost effective and has limitations, such as high-cost fabrication of nanoparticles, low batch-to-batch consistency, high complexity, and relatively low specificity. Reproducible methodologies suitable for the mass production of testing kits based on gold or silver SERS nanotags also remain a challenge. Additionally, the majority of published multiplexed SERS imaging techniques require specific equipment, thus it would be complicated to adapt these methods for clinical needs. Despite these challenges, the field of multiplex SERS has great potential for innovation in diagnostic applications.

\subsection{Plasmon-Enhanced Multiplexed Biosensing}

Plasmon-enhanced fluorescence (PEF) is a phenomenon by which the fluorescence intensity of a nearby fluorophore can be remarkably enhanced by a plasmonic nanostructure [77]. By utilising PEF, the quantum efficiency and photostability of fluorophores are increased. Additionally, extremely low amounts of fluorophore could be detected. Thus, these features enable sensitive detection for very low abundance biomarkers. For the past few years, PEF-based biosensors have received a great deal of attention for the ultrasensitive detection of single analytes. Ventura et al. used the surface of patterned AuNPs for FITC fluorescence enhancement to detect immunoglobulins in real urine samples and showed that their method works in a 10-100 $\mu \mathrm{g} / \mathrm{L}$ detection range with a limit of detection of $8 \mu \mathrm{g} / \mathrm{L}$ [78]. Zhang et al. used an Au nanohole array for prostate-specific antigen detection and demonstrated a limit of detection of $140 \mathrm{fM}$ [79].

Wang et al. demonstrated thrombin and platelet-derived growth factor-BB (PDGF-BB) simultaneous detection using aptamer-modified AgNPs as a capture substrate, and fluorescent dye-modified aptamers as detection probes in a sandwich immunoassay [80]. The linear range of 
thrombin detection was from $55.6 \mathrm{pM}$ to $13.5 \mathrm{nM}$, with the limit of $6.2 \mathrm{pM}$. PDGF-BB was detected with the range of concentration from $625 \mathrm{pM}$ to $20 \mathrm{nM}$ and the detection limit was $156 \mathrm{pM}$. They showed that by using AgNPs probes for $\mathrm{Cy} 3$ and $\mathrm{Cy} 5$ fluorescence enhancement, the detection limit could be improved 80-fold for thrombin and 8-fold for PDGF-BB, when compared to aptamers without enhancement [80].

Liu et al. developed a multiplexed antibody microarray for circulating biomarkers associated with lung cancer detection [81]. Microarray plates were modified with gold nanostructures to enhance the fluorescence of IRDye800, which was used to label detection antibodies in sandwich immunoassay. Lung cancer biomarkers CEA, CYFRA 21-1 and NSE were detected directly from human serum. Simultaneous detection of three biomarkers was achieved by printing capture antibodies onto $3 \times 3$ spot matrices. This approach enables a high-throughput testing of lung cancer biomarkers and improved specificity and sensitivity compared with convenient methods such as ELISA and Luminex assay [81]. Similar studies published by the same group demonstrated detection of diabetes biomarkers [82] as well as zika and dengue viruses [83].

Min et al. demonstrated a new and simple technique that allows the multiplexed detection of biomarkers in extracellular vesicles [84]. In this study, plasmon-enhancement was achieved by using Au nanoholes as a substrate. Firstly, vesicles were captured on Au nanoholes by a biotin-avidin reaction. Then, vesicles were stained with antibodies conjugated with fluorescent labels (Alexa Fluor 488, Cy3, $\mathrm{Cy5}, \mathrm{Cy5.5).} \mathrm{Au} \mathrm{nanoholes} \mathrm{operated} \mathrm{as} \mathrm{amplifiers} \mathrm{for} \mathrm{fluorophores} \mathrm{under} \mathrm{excitation.} \mathrm{They} \mathrm{applied}$ the designed assay to detect glioblastoma biomarkers CD-pan (CD9, CD63, and CD81), with EGFR, EGFRvIII and GAPDH as control markers, from supernatants of glioblastoma cells. Fluorescence signals of multiple fluorophores were amplified by one order, and both transmembrane and intravesicular biomarkers were detected at the single extracellular vesicle level. However, the proposed assay still has limited multiplexing capability, which is dependent on fluorescent microscope systems, allowing the visualisation of up to three or four fluorophores in one sample. Additionally, this system was not tested with clinical samples, thus its real applicability still is unknown [84].

Liu et al. developed an assay for the detection of SARS-CoV-2 antibodies by using nanostructured plasmonic gold substrate as a near-infrared fluorescence amplifier [85]. In this study, IgG and IgM antibodies against SARS-CoV-2 were detected directly from human serum and saliva using a sandwich immunoassay in microarray plate. They demonstrated high specificity and sensitivity for the detection of antibodies against SARS-CoV-2. Additionally, antibodies to viruses associated with common colds did not cross-react with SARS-CoV-2 or SARS, or reaction was low. Application of this fast and sensitive testing method in daily clinical practice would allow population-based diagnostic mass screening of COVID-19 [85].

\section{Upconverting Nanoparticles}

Low background signal and high specific signals from molecules under investigation are two main criteria for precise and effective spectral encoding for multiplexed analysis of various biosamples [86]. The main drawback of previously described methods is interference between coded signals and reporter signals at low concentrations, which leads to inaccurate test results. Nanoparticles are mostly optically active in UV-VIS region, where many biomolecules also show strong autofluorescence (Figure 6a). In this case, upconverting nanoparticles (UCNPs) could be useful for in vivo biosensing. UCNPs are inorganic nanocrystals, commonly rare-earth-doped nanoparticles (RENPs), that allow for the generation of anti-Stokes emission under near-infrared (NIR) excitation. Upconversion (UC) emission itself is a unique process, where low energy NIR light is converted into higher energy light through the sequential absorption of multiple photons or energy transfer. UCNPs exhibit great properties with sharp emission bands, large anti-Stokes shift, high resistance to photobleaching, long lifetimes $(\sim \mathrm{ms})$, high detection sensitivity, low toxicity, and no interference from biomolecular autofluorescence [87] (Figure 6b). The popularity of UCNP application in various biomedical research is constantly growing, including the development of nanobiosensors. 
(a)

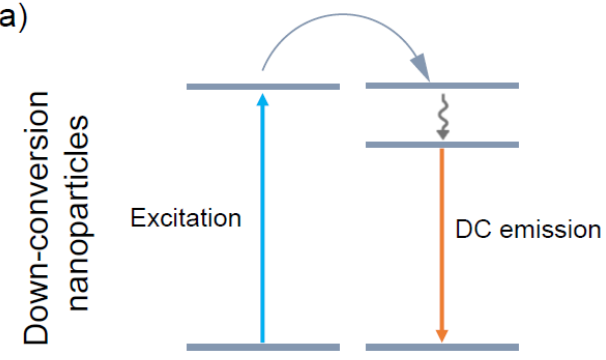

(b)

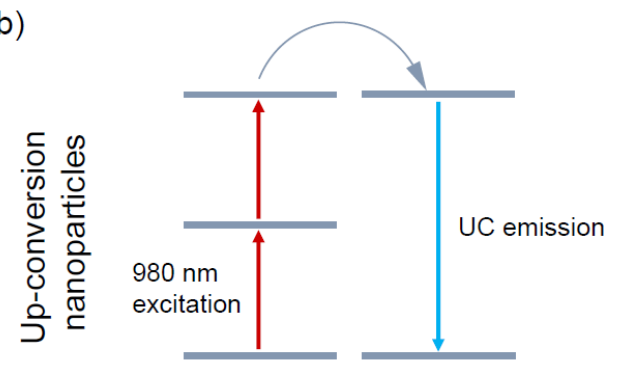

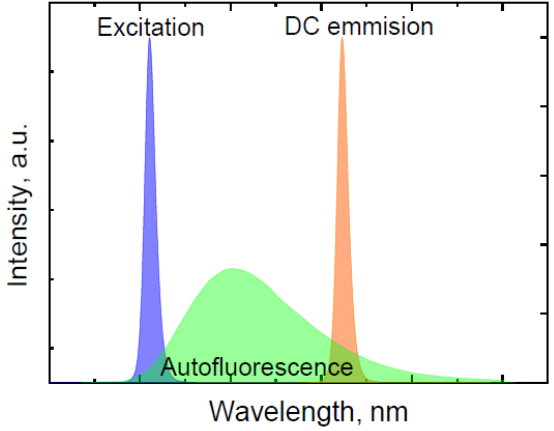

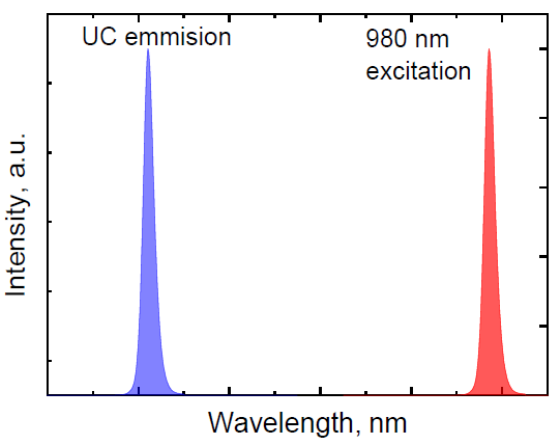

Figure 6. Schematic illustrations of underling luminescence mechanisms: (a) down-conversion nanoparticles are excited in the region where various biomolecules also could be excited and the emission of nanoparticles could overlap with autofluorescence; (b) up-conversion nanoparticles are excited in NIR region where biomolecules are not excited, thus there is no autofluorescence of the sample.

Gorris et al. described UCNPs as background-free codes for multiplexed analyses [86]. In the system proposed by the authors, the surface of the UCNPs $\left(\mathrm{NaYF}_{4}: \mathrm{Yb}, \mathrm{Er}\right.$ and $\left.\mathrm{NaYF}_{4}: \mathrm{Yb}, \mathrm{Tm}\right)$ is modified with a screen layer which consists of different amounts of an organic dye. The absorption spectrum of this dye overlaps with one of the UCNP bands, thus the emission from this band is re-absorbed to different levels by the organic dye. Another emission band is used as a reference. These UCNPs and organic dye complexes could be used as coding elements which provide ratiometric codes in multiplexed system [88].

Zhang et al. created upconversion nanobarcodes (UPNBs)-uniform $\mathrm{Y}_{2} \mathrm{O}_{3}: \mathrm{Yb}, \mathrm{Ho}, \mathrm{Tm} @ \mathrm{Y}_{2} \mathrm{O}_{3} @ \mathrm{SiO}_{2}(\mathrm{COO}-)$ core/shell nanoparticles, which could be used for optical encoding in multiplexed immunoassays [89]. The authors demonstrated that by changing the composition of nanoparticles, several different UPNBs can be created because each nanobarcode has an individual spectrum. Additionally, in order to test synthesised nanobarcodes, UPNBs were conjugated with mouse and rabbit antibodies for two-plexed immunoassays. Additionally, microspheres modified with goat anti-mouse and goat anti-rabbit antibodies (both cascade blue-labelled) were used for the immunodetection of potential receptors. In this way, the recognition process was simulated in the model system. Specific binding between primary antibodies conjugated to UPNBs and secondary antibodies conjugated to microspheres were observed, thus providing proof-of-principle of this model immunoassay [89].

Zhang et al. designed upconverting nanocrystals encoded magnetic microspheres (UCNMMs), which have the potential application for fast separation and multiplexed immunoassays [90]. Six unique upconverting nanocrystals with different upconversion emission spectra were synthesised by a solvent-thermal process. Both upconverting nanocrystals and magnetic nanoparticles were encapsulated into porous poly(styrene-co-EGDMA-co-MAA) beads to obtain UCNMMs. Later UCNMMs were conjugated with goat anti-mouse IgG. For the purpose of detection, FITC-labelled rabbit anti-mouse and IgG PE-labelled goat anti-rabbit IgG antibodies were used. The immunoassay results demonstrated no interrelation between upconversion emission, which was used for encoding, 
and fluorescence of dyes (FITC, PE), which was used as a reporter. Thus, the authors demonstrated the advantage of upconverting nanomaterials' utilisation as barcodes in multiplexed immunoassays compared with traditional down-conversion materials (fluorescent dyes or QDs). The detection limit of mouse IgG in this model immunoassay was $0.01 \mathrm{ng} / \mathrm{mL}$. Thus, their proposed detection system has huge potential applications in multiplexed immunoassays [90].

In order to improve the diagnostic detection of several viruses such as influenza (A and B), respiratory syncytial virus, and adenovirus, Kazakova et al. developed a multiplex serological microarray immunoassay for the simultaneous detection of serum IgG antibodies against these viruses [91]. Microarray plates were coated with streptavidin and the serum antigens as well as negative and positive controls for the samples. Different locations of each of the spotted antigens allowed them to implement specificity of this immunoassay. For detection purposes, anti-human IgG-coated $\mathrm{NaYF}_{4}: \mathrm{Yb}$,Er upconverting nanoparticles were used. UCNPs were chosen to avoid autofluorescence signals from the sample and to increase detection sensitivity. The nanobiosensor was effectively used for the simultaneous detection of antibodies against seven different viruses. The authors suggest that their multiplexed immunoassay is a promising tool for the diagnostic detection of viral infections in serum samples, and can be utilised in epidemiological and seroprevalence studies [91].

Li et al. demonstrated multiplexed upconversion imaging in vivo by registering distinct lifetimes of UCNPs after excitation at $808 \mathrm{~nm}$ [92]. The authors used an $\mathrm{NaYF}_{4} @ \mathrm{NaYbF}_{4} @ \mathrm{NaYF}_{4}: \mathrm{Yb}_{\mathrm{Tm}} @ \mathrm{NaYF}_{4}$ UCNP to control the NIR upconversion luminescence (UCL) lifetime in the range 78-2157 $\mu$ s detected at $808 \mathrm{~nm}$. They conducted in vivo multiplexing experiments using these UCNPs with tuneable lifetimes for in vivo imaging. For the temporal multiplexed imaging, UCNPs with three different lifetimes were injected into a Kunming mouse through tail vein injection and implemented subcutaneous injection into the left and right of the abdomen. UCL signals with two different lifetimes were observed in liver and abdomen subcutis, which corresponded well with UCL imaging. The authors suggest that their temporal upconversion application has the potential for use as a new optical multiplexed imaging technique [92].

Several studies have demonstrated application of RENPs for multiplexed in vivo imaging by utilising only down-conversion emission and lifetime imaging [93-95]. Fan et al. (2018) designed RENPs barcodes with different lifetime characteristics for multiplexed breast cancer biomarkers (ER, PR, HER2) detection in tumour bearing mice [93]. The expression of tumour biomarkers was quantified by lifetime imaging, and a recognition algorithm was used to resolve subtype of tumour, depending on the different expression of biomarkers.

\section{Conclusions}

In this review article, we have provided a comprehensive overview of the current state-of-the-art development of multiplexed nanobiosensors. We have paid special attention to how different nanomaterials could help to achieve better sensitivity and specificity in classical bioassays. The advantages are anticipated in the development of ELISA, flow cytometric, electrochemical and immunofluorescence techniques, with the combination of nanomaterials such as quantum dots, magnetic beads, gold, silver and upconverting nanoparticles. These nanomaterials applied in rapid and cost-effective multiplexed nanobiosensors show promise for the specific and highly sensitive detection of clinically relevant biomarkers. However, there are still some challenges that remain before nanobiosensors can be applied for daily diagnostic purposes. Firstly, colloidal stability of nanoparticles is usually not sufficient for longer shelf life. This disadvantage could be eliminated when nanoparticles are dispersed on substrates by chemical entrapment or by growing them directly on substrates. Another big issue is the biofunctionalisation of nanomaterials. Despite the availability of a broad variety of chemical methods of biomolecule attachment to the surface of nanoparticles, this process usually requires a controlled environment for long-term stability. Repeatability and sensitivity are also key factors for translation from laboratory to clinic. Laboratories are equipped with exclusive and specific equipment, which allows investigations to reach greater sensitivity in 
the developed assays. However, in clinical practice, such equipment would be expensive and time consuming. Nevertheless, several research projects are concentrating on developing simple, cheap and quick assays, suitable for clinical translation and wider implementation in the operational environment of the clinic. Various advantageous aspects of nanomaterials used in multiplexed nanobiosensors are summarised in Table 1, and current evidence implies that these nanomaterials could be used for the development of highly sensitive multiplexed biosensors.

Table 1. Summary of described multiplexing nanobiosensors: used nanoparticles, method of detection, number of detected analytes, detection range and detection limit (if provided).

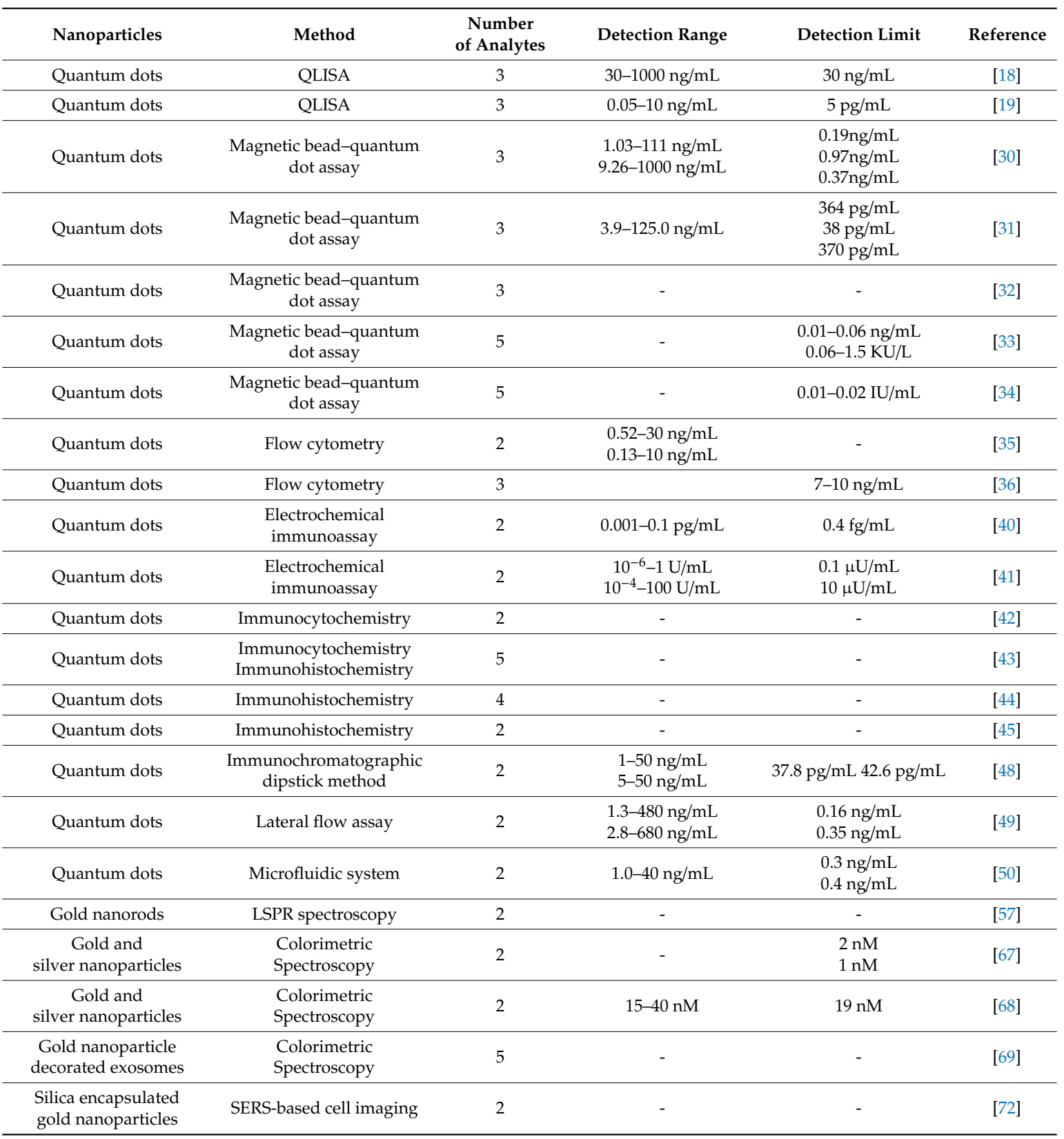


Table 1. Cont.

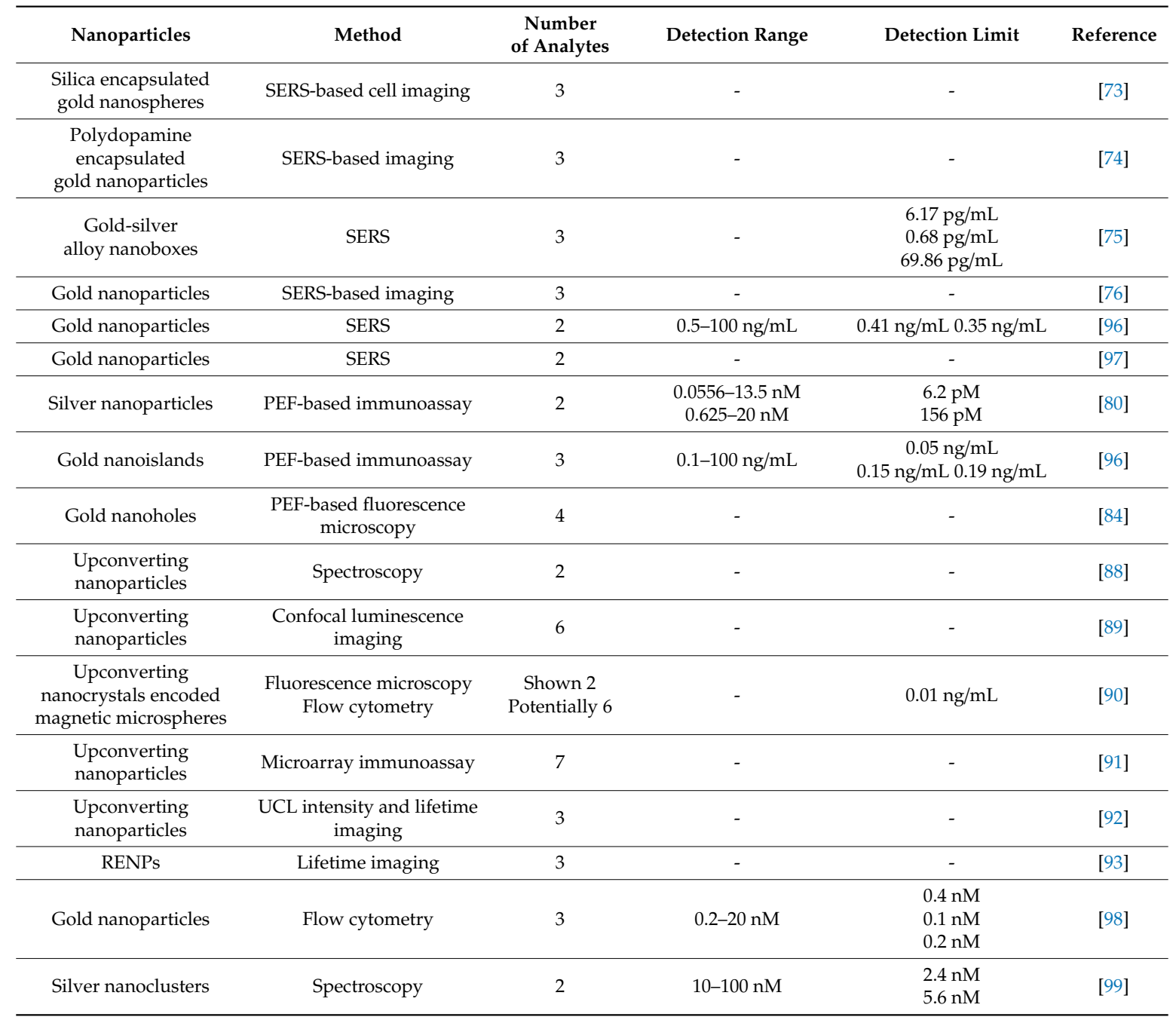

Author Contributions: Writing—original draft preparation, G.J.; writing—review and editing, V.K.; visualisation, G.J. and V.K.; supervision, R.R. and A.M.; funding acquisition, A.M. All authors have read and agreed to the published version of the manuscript.

Funding: This research was funded by the European Regional Development Fund through the Research Council of Lithuania according to the Programme Attracting Foreign Researchers for Research Implementation, Grant No. 01.2.2-LMT-K-718-02-0022.

Conflicts of Interest: The authors declare no conflict of interest.

\section{References}

1. Song, Y.; Huang, Y.-Y.; Liu, X.; Zhang, X.; Ferrari, M.; Qin, L. Point-of-care technologies for molecular diagnostics using a drop of blood. Trends Biotechnol. 2014, 32, 132-139. [CrossRef] [PubMed]

2. Nimse, S.B.; Sonawane, M.D.; Song, K.-S.; Kim, T. Biomarker detection technologies and future directions. Analyst 2016, 141, 740-755. [CrossRef] [PubMed]

3. Kosaka, N.; Kogure, A.; Yamamoto, T.; Urabe, F.; Usuba, W.; Prieto-Vila, M.; Ochiya, T. Exploiting the message from cancer: The diagnostic value of extracellular vesicles for clinical applications. Exp. Mol. Med. 2019, 51, 1-9. [CrossRef] [PubMed]

4. Fan, Y.; Wang, S.; Zhang, F. Optical Multiplexed Bioassays for Improved Biomedical Diagnostics. Angew. Chem. Int. Ed. 2019, 58, 13208-13219. [CrossRef] 
5. Elsabahy, M.; Wooley, K.L.; Hendricksen, A.; Oh, K. Multiplexing techniques for measurement of the immunomodulatory effects of particulate materials: Precautions when testing micro- and nano-particles. Methods 2019, 158, 81-85. [CrossRef]

6. Bruchez, M.; Moronne, M.; Gin, P.; Weiss, S.; Alivisatos, A.P. Semiconductor nanocrystals as fluorescent biological labels. Science 1998, 281, 2013-2016. [CrossRef]

7. Alivisatos, A.P. Semiconductor Clusters, Nanocrystals, and Quantum Dots. Science 1996, 271, $933-937$. [CrossRef]

8. Chan, W.C.W.; Maxwell, D.J.; Gao, X.; Bailey, R.E.; Han, M.; Nie, S. Luminescent quantum dots for multiplexed biological detection and imaging. Curr. Opin. Biotechnol. 2002, 13, 40-46. [CrossRef]

9. Bilan, R.; Fleury, F.; Nabiev, I.; Sukhanova, A. Quantum Dot Surface Chemistry and Functionalization for Cell Targeting and Imaging. Bioconjug. Chem. 2015, 26, 609-624. [CrossRef]

10. Suzuki, M.; Udaka, H.; Fukuda, T. Quantum dot-linked immunosorbent assay (QLISA) using orientation-directed antibodies. J. Pharm. Biomed. Anal. 2017, 143, 110-115. [CrossRef]

11. Powers, A.D.; Palecek, S.P. Protein analytical assays for diagnosing, monitoring, and choosing treatment for cancer patients. J. Healthc. Eng. 2012, 3, 503-534. [CrossRef] [PubMed]

12. Satija, J.; Punjabi, N.; Mishra, D.; Mukherji, S. Plasmonic-ELISA: Expanding horizons. RSC Adv. 2016, 6, 85440-85456. [CrossRef]

13. Thaxton, C.S.; Nam, J.-M.; Mirkin, C.A. PCR-like sensitivity for proteins with bio-bar-code amplification. Discov. Med. 2003, 3, 58-60. [PubMed]

14. Elshal, M.F.; McCoy, J.P. Multiplex bead array assays: Performance evaluation and comparison of sensitivity to ELISA. Methods 2006, 38, 317-323. [CrossRef]

15. Lakowicz, J.R. (Ed.) Fluorescence Sensing. In Principles of Fluorescence Spectroscopy; Springer: Boston, MA, USA, 2006; pp. 623-673.

16. Grabolle, M.; Spieles, M.; Lesnyak, V.; Gaponik, N.; Eychmüller, A.; Resch-Genger, U. Determination of the Fluorescence Quantum Yield of Quantum Dots: Suitable Procedures and Achievable Uncertainties. Anal. Chem. 2009, 81, 6285-6294. [CrossRef]

17. Mansur, H.S.; Mansur, A.A.P.; Soriano-Araújo, A.; Lobato, Z.I.P.; de Carvalho, S.M.; de Fatima Leite, M. Water-soluble nanoconjugates of quantum dot-chitosan-antibody for in vitro detection of cancer cells based on "enzyme-free" fluoroimmunoassay. Mater. Sci. Eng. C Mater. Biol. Appl. 2015, 52, 61-71. [CrossRef]

18. Goldman, E.R.; Clapp, A.R.; Anderson, G.P.; Uyeda, H.T.; Mauro, J.M.; Medintz, I.L.; Mattoussi, H. Multiplexed toxin analysis using four colors of quantum dot fluororeagents. Anal. Chem. 2004, 76, 684-688. [CrossRef]

19. Song, E.; Yu, M.; Wang, Y.; Hu, W.; Cheng, D.; Swihart, M.T.; Song, Y. Multi-color quantum dot-based fluorescence immunoassay array for simultaneous visual detection of multiple antibiotic residues in milk. Biosens. Bioelectron. 2015, 72, 320-325. [CrossRef]

20. Kim, C.; Searson, P.C. Magnetic bead-quantum dot assay for detection of a biomarker for traumatic brain injury. Nanoscale 2015, 7, 17820-17826. [CrossRef]

21. Horan, P.K.; Wheeless, L.L. Quantitative single cell analysis and sorting. Science 1977, 198, 149-157. [CrossRef]

22. Leng, S.X.; McElhaney, J.E.; Walston, J.D.; Xie, D.; Fedarko, N.S.; Kuchel, G.A. ELISA and multiplex technologies for cytokine measurement in inflammation and aging research. J. Gerontol. A Biol. Sci. Med. Sci. 2008, 63, 879-884. [CrossRef] [PubMed]

23. Kass, D.J.; Nouraie, M.; Glassberg, M.K.; Ramreddy, N.; Fernandez, K.; Harlow, L.; Zhang, Y.; Chen, J.; Kerr, G.S.; Reimold, A.M.; et al. Comparative Profiling of Serum Protein Biomarkers in Rheumatoid Arthritis-Associated Interstitial Lung Disease and Idiopathic Pulmonary Fibrosis. Arthritis Rheumatol. 2020, 72, 409-419. [CrossRef] [PubMed]

24. Yu, F.; Xiong, Y.-M.; Yu, S.-C.; He, L.-L.; Niu, S.-S.; Wu, Y.-M.; Liu, J.; Qu, L.-B.; Liu, L.-E.; Wu, Y.-J. Magnetic immunoassay using CdSe/ZnS quantum dots as fluorescent probes to detect the level of DNA methyltransferase 1 in human serum sample. Int. J. Nanomed. 2018, 13, 429-437. [CrossRef] [PubMed]

25. Faresjö, M. A useful guide for analysis of immune markers by fluorochrome (Luminex) technique. Methods Mol. Biol. 2014, 1172, 87-96. [CrossRef]

26. Zhu, X.; Duan, D.; Publicover, N.G. Magnetic bead based assay for C-reactive protein using quantum-dot fluorescence labeling and immunoaffinity separation. Analyst 2010, 135, 381-389. [CrossRef] 
27. Park, H.; Hwang, M.P.; Lee, J.-W.; Choi, J.; Lee, K.H. Harnessing immunomagnetic separation and quantum dot-based quantification capacities for the enumeration of absolute levels of biomarker. Nanotechnology 2013, 24, 285103. [CrossRef]

28. Park, H.; Lee, J.-W.; Hwang, M.P.; Lee, K.H. Quantification of cardiovascular disease biomarkers via functionalized magnetic beads and on-demand detachable quantum dots. Nanoscale 2013, 5, 8609-8615. [CrossRef]

29. Kim, C.; Hoffmann, G.; Searson, P.C. Integrated Magnetic Bead-Quantum Dot Immunoassay for Malaria Detection. ACS Sens. 2017, 2, 766-772. [CrossRef]

30. Liu, L.; Wu, S.; Jing, F.; Zhou, H.; Jia, C.; Li, G.; Cong, H.; Jin, Q.; Zhao, J. Bead-based microarray immunoassay for lung cancer biomarkers using quantum dots as labels. Biosens. Bioelectron. 2016, 80, 300-306. [CrossRef]

31. Wu, S.; Liu, L.; Li, G.; Jing, F.; Mao, H.; Jin, Q.; Zhai, W.; Zhang, H.; Zhao, J.; Jia, C. Multiplexed detection of lung cancer biomarkers based on quantum dots and microbeads. Talanta 2016, 156-157, 48-54. [CrossRef]

32. Bai, Y.; Lu, Y.; Wang, K.; Cheng, Z.; Qu, Y.; Qiu, S.; Zhou, L.; Wu, Z.; Liu, H.; Zhao, J.; et al. Rapid Isolation and Multiplexed Detection of Exosome Tumor Markers Via Queued Beads Combined with Quantum Dots in a Microarray. Nano-Micro Lett. 2019, 11, 59. [CrossRef]

33. Leng, Y.; Wu, W.; Li, L.; Lin, K.; Sun, K.; Chen, X.; Li, W. Magnetic/Fluorescent Barcodes Based on Cadmium-Free Near-Infrared-Emitting Quantum Dots for Multiplexed Detection. Adv. Funct. Mater. 2016, 26, 7581-7589. [CrossRef]

34. Wu, W.; Yu, X.; Gao, M.; Gull, S.; Shen, L.; Wang, W.; Li, L.; Yin, Y.; Li, W. Precisely Encoded Barcodes Using Tetrapod CdSe/CdS Quantum Dots with a Large Stokes Shift for Multiplexed Detection. Adv. Funct. Mater. 2020, 30, 1906707. [CrossRef]

35. Yu, H.-W.; Kim, I.S.; Niessner, R.; Knopp, D. Multiplex competitive microbead-based flow cytometric immunoassay using quantum dot fluorescent labels. Anal. Chim. Acta 2012, 750, 191-198. [CrossRef] [PubMed]

36. Bilan, R.; Ametzazurra, A.; Brazhnik, K.; Escorza, S.; Fernández, D.; Uríbarri, M.; Nabiev, I.; Sukhanova, A. Quantum-dot-based suspension microarray for multiplex detection of lung cancer markers: Preclinical validation and comparison with the Luminex xMAP ${ }^{\circledR}$ system. Sci. Rep. 2017, 7, 1-10. [CrossRef] [PubMed]

37. Fowler, J.M.; Wong, D.K.Y.; Halsall, H.B.; Heineman, W.R. Recent developments in electrochemical immunoassays and immunosensors. In Electrochemical Sensors, Biosensors and their Biomedical Applications; Academic Press: Amsterdam, The Netherlands; Boston, MA, USA, 2008; pp. 115-143. [CrossRef]

38. Wang, J.; Liu, G.; Polsky, R.; Merkoçi, A. Electrochemical stripping detection of DNA hybridization based on cadmium sulfide nanoparticle tags. Electrochem. Commun. 2002, 4, 722-726. [CrossRef]

39. Wang, J.; Liu, G.; Merkoçi, A. Electrochemical coding technology for simultaneous detection of multiple DNA targets. J. Am. Chem. Soc. 2003, 125, 3214-3215. [CrossRef]

40. Guo, Z.; Hao, T.; Du, S.; Chen, B.; Wang, Z.; Li, X.; Wang, S. Multiplex electrochemiluminescence immunoassay of two tumor markers using multicolor quantum dots as labels and graphene asconductingbridge. Biosens. Bioelectron. 2013, 44, 101-107. [CrossRef]

41. Babamiri, B.; Hallaj, R.; Salimi, A. Ultrasensitive electrochemiluminescence immunoassay for simultaneous determination of CA125 and CA15-3 tumor markers based on PAMAM-sulfanilic acid-Ru(bpy)32+ and PAMAM-CdTe@CdS nanocomposite. Biosens. Bioelectron. 2018, 99, 353-360. [CrossRef]

42. Wu, X.; Liu, H.; Liu, J.; Haley, K.N.; Treadway, J.A.; Larson, J.P.; Ge, N.; Peale, F.; Bruchez, M.P. Immunofluorescent labeling of cancer marker Her2 and other cellular targets with semiconductor quantum dots. Nat. Biotechnol. 2003, 21, 41-46. [CrossRef]

43. Yezhelyev, M.V.; Al-Hajj, A.; Morris, C.; Marcus, A.I.; Liu, T.; Lewis, M.; Cohen, C.; Zrazhevskiy, P.; Simons, J.W.; Rogatko, A.; et al. In Situ Molecular Profiling of Breast Cancer Biomarkers with Multicolor Quantum Dots. Adv. Mater. 2007, 19, 3146-3151. [CrossRef]

44. Liu, J.; Lau, S.K.; Varma, V.A.; Kairdolf, B.A.; Nie, S. Multiplexed Detection and Characterization of Rare Tumor Cells in Hodgkin's Lymphoma with Multicolor Quantum Dots. Anal. Chem. 2010, 82, 6237-6243. [CrossRef] [PubMed]

45. Xu, H.; Xu, J.; Wang, X.; Wu, D.; Chen, Z.G.; Wang, A.Y. Quantum Dot-Based, Quantitative, and Multiplexed Assay for Tissue Staining. ACS Appl. Mater. Interfaces 2013, 5, 2901-2907. [CrossRef] [PubMed] 
46. Kuswandi, B.; Ensafi, A.A. Perspective-Paper-Based Biosensors: Trending Topic in Clinical Diagnostics Developments and Commercialization. J. Electrochem. Soc. 2019, 167, 037509. [CrossRef]

47. Chinnadayyala, S.R.; Park, J.; Le, H.T.N.; Santhosh, M.; Kadam, A.N.; Cho, S. Recent advances in microfluidic paper-based electrochemiluminescence analytical devices for point-of-care testing applications. Biosens. Bioelectron. 2019, 126, 68-81. [CrossRef]

48. Xiao, K.; Wang, K.; Qin, W.; Hou, Y.; Lu, W.; Xu, H.; Wo, Y.; Cui, D. Use of quantum dot beads-labeled monoclonal antibody to improve the sensitivity of a quantitative and simultaneous immunochromatographic assay for neuron specific enolase and carcinoembryonic antigen. Talanta 2017, 164, 463-469. [CrossRef]

49. Chen, Z.; Liang, R.; Guo, X.; Liang, J.; Deng, Q.; Li, M.; An, T.; Liu, T.; Wu, Y. Simultaneous quantitation of cytokeratin-19 fragment and carcinoembryonic antigen in human serum via quantum dot-doped nanoparticles. Biosens. Bioelectron. 2017, 91, 60-65. [CrossRef]

50. Chen, Y.; Guo, X.; Liu, W.; Zhang, L. Paper-based fluorometric immunodevice with quantum-dot labeled antibodies for simultaneous detection of carcinoembryonic antigen and prostate specific antigen. Microchim. Acta 2019, 186, 112. [CrossRef]

51. Jain, P.K.; Huang, X.; El-Sayed, I.H.; El-Sayed, M.A. Noble Metals on the Nanoscale: Optical and Photothermal Properties and Some Applications in Imaging, Sensing, Biology, and Medicine. Acc. Chem. Res. 2008, 41, 1578-1586. [CrossRef]

52. Tao, Y.; Li, M.; Ren, J.; Qu, X. Metal nanoclusters: Novel probes for diagnostic and therapeutic applications. Chem. Soc. Rev. 2015, 44, 8636-8663. [CrossRef]

53. Elahi, N.; Kamali, M.; Baghersad, M.H. Recent biomedical applications of gold nanoparticles: A review. Talanta 2018, 184, 537-556. [CrossRef] [PubMed]

54. Jeong, H.-H.; Choi, E.; Ellis, E.; Lee, T.-C. Recent advances in gold nanoparticles for biomedical applications: From hybrid structures to multi-functionality. J. Mater. Chem. B 2019, 7, 3480-3496. [CrossRef]

55. Le Ouay, B.; Stellacci, F. Antibacterial activity of silver nanoparticles: A surface science insight. Nano Today 2015, 10, 339-354. [CrossRef]

56. Moores, A.; Goettmann, F. The plasmon band in noble metal nanoparticles: An introduction to theory and applications. New J. Chem. 2006, 30, 1121-1132. [CrossRef]

57. Huang, H.; Liu, F.; Huang, S.; Yuan, S.; Liao, B.; Yi, S.; Zeng, Y.; Chu, P.K. Sensitive and simultaneous detection of different disease markers using multiplexed gold nanorods. Anal. Chim. Acta 2012, 755, 108-114. [CrossRef]

58. Liang, J.; Yao, C.; Li, X.; Wu, Z.; Huang, C.; Fu, Q.; Lan, C.; Cao, D.; Tang, Y. Silver Nanoprism Etching-Based Plasmonic ELISA for the High Sensitive Detection of Prostate-Specific Antigen. Biosens Bioelectron 2015, 69, 128-134. [CrossRef]

59. Liu, D.; Yang, J.; Wang, H.-F.; Wang, Z.; Huang, X.; Wang, Z.; Niu, G.; Hight Walker, A.R.; Chen, X. Glucose oxidase-catalyzed growth of gold nanoparticles enables quantitative detection of attomolar cancer biomarkers. Anal. Chem. 2014, 86, 5800-5806. [CrossRef]

60. Rodríguez-Lorenzo, L.; de la Rica, R.; Álvarez-Puebla, R.A.; Liz-Marzán, L.M.; Stevens, M.M. Plasmonic nanosensors with inverse sensitivity by means of enzyme-guided crystal growth. Nat. Mater. 2012, 11, 604-607. [CrossRef]

61. Nie, X.-M.; Huang, R.; Dong, C.-X.; Tang, L.-J.; Gui, R.; Jiang, J.-H. Plasmonic ELISA for the ultrasensitive detection of Treponema pallidum. Biosens. Bioelectron. 2014, 58, 314-319. [CrossRef]

62. Mohd Bakhori, N.; Yusof, N.A.; Abdullah, J.; Wasoh, H.; Md Noor, S.S.; Ahmad Raston, N.H.; Mohammad, F. Immuno Nanosensor for the Ultrasensitive Naked Eye Detection of Tuberculosis. Sensors 2018, 18, 1932. [CrossRef]

63. Peng, M.-P.; Ma, W.; Long, Y.-T. Alcohol Dehydrogenase-Catalyzed Gold Nanoparticle Seed-Mediated Growth Allows Reliable Detection of Disease Biomarkers with the Naked Eye. Anal. Chem. 2015, 87, 5891-5896. [CrossRef] [PubMed]

64. Cecchin, D.; de la Rica, R.; Bain, R.E.S.; Finnis, M.W.; Stevens, M.M.; Battaglia, G. Plasmonic ELISA for the detection of gp120 at ultralow concentrations with the naked eye. Nanoscale 2014, 6, 9559-9562. [CrossRef] [PubMed]

65. Chang, C.-C.; Chen, C.-P.; Wu, T.-H.; Yang, C.-H.; Lin, C.-W.; Chen, C.-Y. Gold Nanoparticle-Based Colorimetric Strategies for Chemical and Biological Sensing Applications. Nanomaterials 2019, 9, 861. [CrossRef] [PubMed] 
66. Sabela, M.; Balme, S.; Bechelany, M.; Janot, J.-M.; Bisetty, K. A Review of Gold and Silver Nanoparticle-Based Colorimetric Sensing Assays. Adv. Eng. Mater. 2017, 19, 1700270. [CrossRef]

67. Mancuso, M.; Jiang, L.; Cesarman, E.; Erickson, D. Multiplexed Colorimetric Detection of Kaposi's Sarcoma Associated Herpesvirus and Bartonella DNA using Gold and Silver Nanoparticles. Nanoscale 2013, 5, 1678-1686. [CrossRef]

68. Heo, J.H.; Yi, G.S.; Lee, B.S.; Cho, H.H.; Lee, J.W.; Lee, J.H. A significant enhancement of color transition from an on-off type achromatic colorimetric nanosensor for highly sensitive multi-analyte detection with the naked eye. Nanoscale 2016, 8, 18341-18351. [CrossRef]

69. Di, H.; Mi, Z.; Sun, Y.; Liu, X.; Liu, X.; Li, A.; Jiang, Y.; Gao, H.; Rong, P.; Liu, D. Nanozyme-assisted sensitive profiling of exosomal proteins for rapid cancer diagnosis. Theranostics 2020, 10, 9303-9314. [CrossRef]

70. Lee, H.; Gao, X.; Kim, Y.-P. Immuno-Nanoparticles for Multiplex Protein Imaging in Cells and Tissues. BioChip J. 2018, 12, 83-92. [CrossRef]

71. Schlücker, S. Surface-enhanced Raman spectroscopy: Concepts and chemical applications. Angew. Chem. Int. Ed. 2014, 53, 4756-4795. [CrossRef]

72. Lee, S.; Chon, H.; Yoon, S.-Y.; Lee, E.K.; Chang, S.-I.; Lim, D.W.; Choo, J. Fabrication of SERS-fluorescence dual modal nanoprobes and application to multiplex cancer cell imaging. Nanoscale 2012, 4, 124-129. [CrossRef]

73. Lee, S.; Chon, H.; Lee, J.; Ko, J.; Chung, B.H.; Lim, D.W.; Choo, J. Rapid and sensitive phenotypic marker detection on breast cancer cells using surface-enhanced Raman scattering (SERS) imaging. Biosens. Bioelectron. 2014, 51, 238-243. [CrossRef] [PubMed]

74. Sun, C.; Zhang, L.; Zhang, R.; Gao, M.; Zhang, X. Facilely synthesized polydopamine encapsulated surface-enhanced Raman scattering (SERS) probes for multiplex tumor associated cell surface antigen detection using SERS imaging. RSC Adv. 2015, 5, 72369-72372. [CrossRef]

75. Li, J.; Wang, J.; Grewal, Y.S.; Howard, C.B.; Raftery, L.J.; Mahler, S.; Wang, Y.; Trau, M. Multiplexed SERS Detection of Soluble Cancer Protein Biomarkers with Gold-Silver Alloy Nanoboxes and Nanoyeast Single-Chain Variable Fragments. Anal. Chem. 2018, 90, 10377-10384. [CrossRef] [PubMed]

76. Li, M.; Wu, J.; Ma, M.; Feng, Z.; Mi, Z.; Rong, P.; Liu, D. Alkyne- and Nitrile-Anchored Gold Nanoparticles for Multiplex SERS Imaging of Biomarkers in Cancer Cells and Tissues. Nanotheranostics 2019, 3, 113-119. [CrossRef] [PubMed]

77. Fothergill, S.M.; Joyce, C.; Xie, F. Metal enhanced fluorescence biosensing: From ultra-violet towards second near-infrared window. Nanoscale 2018, 10, 20914-20929. [CrossRef] [PubMed]

78. Della Ventura, B.; Gelzo, M.; Battista, E.; Alabastri, A.; Schirato, A.; Castaldo, G.; Corso, G.; Gentile, F.; Velotta, R. Biosensor for Point-of-Care Analysis of Immunoglobulins in Urine by Metal Enhanced Fluorescence from Gold Nanoparticles. ACS Appl. Mater. Interfaces 2019, 11, 3753-3762. [CrossRef]

79. Zhang, Q.; Wu, L.; Wong, T.I.; Zhang, J.; Liu, X.; Zhou, X.; Bai, P.; Liedberg, B.; Wang, Y. Surface plasmon-enhanced fluorescence on Au nanohole array for prostate-specific antigen detection. Int. J. Nanomed. 2017, 12, 2307-2314. [CrossRef]

80. Wang, Y.; Li, H.; Xu, D. Aptamers-based sandwich assay for silver-enhanced fluorescence multiplex detection. Anal. Chim. Acta 2016, 905, 149-155. [CrossRef]

81. Liu, B.; Li, Y.; Wan, H.; Wang, L.; Xu, W.; Zhu, S.; Liang, Y.; Zhang, B.; Lou, J.; Dai, H.; et al. High Performance, Multiplexed Lung Cancer Biomarker Detection on a Plasmonic Gold Chip. Adv. Funct. Mater. 2016, 26, 7994-8002. [CrossRef]

82. Zhang, B.; Kumar, R.B.; Dai, H.; Feldman, B.J. A plasmonic chip for biomarker discovery and diagnosis of type 1 diabetes. Nat. Med. 2014, 20, 948-953. [CrossRef]

83. Zhang, B.; Pinsky, B.A.; Ananta, J.S.; Zhao, S.; Arulkumar, S.; Wan, H.; Sahoo, M.K.; Abeynayake, J.; Waggoner, J.J.; Hopes, C.; et al. Diagnosis of Zika virus infection on a nanotechnology platform. Nat. Med. 2017, 23, 548-550. [CrossRef] [PubMed]

84. Min, J.; Son, T.; Hong, J.-S.; Cheah, P.S.; Wegemann, A.; Murlidharan, K.; Weissleder, R.; Lee, H.; Im, H. Plasmon-Enhanced Biosensing for Multiplexed Profiling of Extracellular Vesicles. Adv. Biosyst. 2020, 2000003. [CrossRef] [PubMed]

85. Liu, T.; Hsiung, J.; Zhao, S.; Kost, J.; Sreedhar, D.; Hanson, C.V.; Olson, K.; Keare, D.; Chang, S.T.; Bliden, K.P.; et al. Quantification of antibody avidities and accurate detection of SARS-CoV-2 antibodies in serum and saliva on plasmonic substrates. Nat. Biomed. Eng. 2020, 1-9. [CrossRef] [PubMed] 
86. Gorris, H.H.; Wolfbeis, O.S. Photon-Upconverting Nanoparticles for Optical Encoding and Multiplexing of Cells, Biomolecules, and Microspheres. Angew. Chem. Int. Ed. 2013, 52, 3584-3600. [CrossRef] [PubMed]

87. Chen, F.; Bu, W.; Cai, W.; Shi, J. Functionalized Upconversion Nanoparticles: Versatile Nanoplatforms for Translational Research. Curr. Mol. Med. 2013, 13, 1613-1632. [CrossRef] [PubMed]

88. Gorris, H.H.; Ali, R.; Saleh, S.M.; Wolfbeis, O.S. Tuning the dual emission of photon-upconverting nanoparticles for ratiometric multiplexed encoding. Adv. Mater. 2011, 23, 1652-1655. [CrossRef] [PubMed]

89. Zhang, F.; Haushalter, R.C.; Haushalter, R.W.; Shi, Y.; Zhang, Y.; Ding, K.; Zhao, D.; Stucky, G.D. Rare Earth Upconverting Nanobarcodes for Multiplexed Biological Detection. Small 2011, 7, 1972-1976. [CrossRef] [PubMed]

90. Zhang, Y.; Dong, C.; Su, L.; Wang, H.; Gong, X.; Wang, H.; Liu, J.; Chang, J. Multifunctional Microspheres Encoded with Upconverting Nanocrystals and Magnetic Nanoparticles for Rapid Separation and Immunoassays. ACS Appl. Mater. Interfaces 2016, 8, 745-753. [CrossRef] [PubMed]

91. Kazakova, A.; Kakkola, L.; Päkkilä, H.; Teros-Jaakkola, T.; Soukka, T.; Peltola, V.; Waris, M.; Julkunen, I. Serological Array-in-Well Multiplex Assay Reveals a High Rate of Respiratory Virus Infections and Reinfections in Young Children. mSphere 2019, 4. [CrossRef] [PubMed]

92. Li, H.; Tan, M.; Wang, X.; Li, F.; Zhang, Y.; Zhao, L.; Yang, C.; Chen, G. Temporal Multiplexed In Vivo Upconversion Imaging. J. Am. Chem. Soc. 2020, 142, 2023-2030. [CrossRef] [PubMed]

93. Fan, Y.; Wang, P.; Lu, Y.; Wang, R.; Zhou, L.; Zheng, X.; Li, X.; Piper, J.A.; Zhang, F. Lifetime-engineered NIR-II nanoparticles unlock multiplexed in vivo imaging. Nat. Nanotechnol. 2018, 13, 941-946. [CrossRef] [PubMed]

94. Zhang, H.; Fan, Y.; Pei, P.; Sun, C.; Lu, L.; Zhang, F. Tm3+-Sensitized NIR-II Fluorescent Nanocrystals for In Vivo Information Storage and Decoding. Angew. Chem. 2019, 131, 10259-10263. [CrossRef]

95. Tian, R.; Ma, H.; Zhu, S.; Lau, J.; Ma, R.; Liu, Y.; Lin, L.; Chandra, S.; Wang, S.; Zhu, X.; et al. Multiplexed NIR-II Probes for Lymph Node-Invaded Cancer Detection and Imaging-Guided Surgery. Adv. Mater. 2020, 32, 1907365. [CrossRef] [PubMed]

96. Li, L.; Liu, C.; Cao, X.; Tan, L.; Lu, W. Multiplexing determination of cancer-associated biomarkers by surface-enhanced Raman scattering using ordered gold nanohoneycomb arrays. Bioanalysis 2017, 9, 1561-1572. [CrossRef] [PubMed]

97. Ou, Y.-C.; Webb, J.A.; O’Brien, C.M.; Pence, I.J.; Lin, E.C.; Paul, E.P.; Cole, D.; Ou, S.-H.; Lapierre-Landry, M.; DeLapp, R.C.; et al. Diagnosis of immunomarkers in vivo via multiplexed surface enhanced Raman spectroscopy with gold nanostars. Nanoscale 2018, 10, 13092-13105. [CrossRef] [PubMed]

98. Li, C.; Li, G.; Liu, H.; Xiao, Z.; Jin, M.; Yuan, C. A novel gold nanoparticles decorated magnetic microbead-based molecular beacon for DNA multiplexing detection by flow cytometry. Anal. Chim. Acta 2020, 1110, 19-25. [CrossRef] [PubMed]

99. Jiang, Y.; Tang, Y.; Miao, P. Polydopamine nanosphere@silver nanoclusters for fluorescence detection of multiplex tumor markers. Nanoscale 2019, 11, 8119-8123. [CrossRef]

Publisher's Note: MDPI stays neutral with regard to jurisdictional claims in published maps and institutional affiliations.

(C) 2020 by the authors. Licensee MDPI, Basel, Switzerland. This article is an open access article distributed under the terms and conditions of the Creative Commons Attribution (CC BY) license (http://creativecommons.org/licenses/by/4.0/). 